\title{
Invariant Manifolds and Their Zero-Viscosity Limits for Navier-Stokes Equations
}

\author{
Y. Charles Li \\ Communicated by Charles Li, received May 1, 2005
}

\begin{abstract}
First we prove a general spectral theorem for the linear NavierStokes (NS) operator in both 2D and 3D. The spectral theorem says that the spectrum consists of only eigenvalues which lie in a parabolic region, and the eigenfunctions (and higher order eigenfunctions) form a complete basis in $H^{\ell}$ $(\ell=0,1,2, \cdots)$. Then we prove the existence of invariant manifolds. We are also interested in a more challenging problem, i.e. studying the zero-viscosity limits $\left(\nu \rightarrow 0^{+}\right)$of the invariant manifolds. Under an assumption, we can show that the sizes of the unstable manifold and the center-stable manifold of a steady state are $O(\sqrt{\nu})$, while the sizes of the stable manifold, the center manifold, and the center-unstable manifold are $O(\nu)$, as $\nu \rightarrow 0^{+}$. Finally, we study three examples. The first example is defined on a rectangular periodic domain, and has only one unstable eigenvalue which is real. A complete estimate on this eigenvalue is obtained. Existence of an 1D unstable manifold and a codim 1 stable manifold is proved without any assumption. For the other two examples, partial estimates on the eigenvalues are obtained.
\end{abstract}

\section{Contents}

1. Introduction 159

2. Formulation of the Problems 161

3. Invariant Manifolds 162

4. Examples 169

References $\quad 185$

\section{Introduction}

Navier-Stokes equations define an infinite dimensional dynamical system. Turbulence can be represented through a chaotic solution in the infinite dimensional phase spaces. For example, one can take the Sobolev spaces $H^{\ell}(\ell=0,1,2, \cdots)$ to

1991 Mathematics Subject Classification. Primary 35, 76, 37; Secondary 34.

Key words and phrases. Invariant manifold, zero-viscosity limit, Navier-Stokes equation. 
be the phase spaces. A basic dynamical system question is: Does the phase space foliate under the NS flow? That is, are there invariant manifolds under the NS flow ? The answer is yes. It seems that under the Euler flow, which can be obtained from the NS flow by setting viscosity to zero, the phase space does not foliate. To begin a dynamical system study, one chooses a common steady state of the NS and its corresponding Euler equation, defined on a periodic spatial domain. At this steady state, the spectra of the linear NS and the linear Euler are dramatically different. The linear NS has the form: $\nu \Delta+$ relatively compact terms, therefore, it has only a point spectrum via Weyl's essential spectrum theorem. On the other hand, the linear Euler has a nontrivial essential spectrum. For 2D linear Euler, if the steady state as a vector field has a saddle, then the essential spectrum in the Sobolev space $H^{\ell}(\ell=0,1,2, \cdots)$ is a vertical band symmetric with respect to the imaginary axis [25]. The width of the band $(=\ell \Lambda$ for some $\Lambda>0)$ increases with $\ell$, representing the fact of cascade and inverse cascade. A typical example of such steady states is the cat's eye. If the steady state has no saddle, but has at least two centers, then the essential spectrum in the Sobolev space $H^{\ell}$ is the imaginary axis $[\mathbf{2 5}]$. A typical example of such steady states is the shear $[\mathbf{1 6}][\mathbf{9}]$. For the shear, a sharp upper bound on the number of eigenvalues was also obtained [8]. For 3D linear Euler, the spectrum is an open problem. A nice example of the steady states is the $\mathrm{ABC}$ flow vector field.

An interesting open problem is to understand the connection between the spectra of linear NS and linear Euler as $\nu \rightarrow 0^{+}$. Besides the progresses mentioned above in understanding the spectra of linear Euler, progresses were also made in better understanding of the spectra of linear NS, although they were in a different setting. For both 2D and 3D NS posed in $L^{2}$ (in terms of weak solutions) on a bounded domain with non-slip boundary condition, Prodi $[\mathbf{2 2}]$ proved that the eigenvalues of the linear NS at a steady state lie inside a parabolic region $\lambda_{r}<a-b \lambda_{i}^{2}$ where $a>0$ and $b>0$ are some constants, and $\lambda=\lambda_{r}+i \lambda_{i}$ is the spectral parameter. Moreover, David Sattinger [24] proved that the linear NS has infinitely many eigenvalues lying in the parabolic region, each of finite multiplicity, which can cluster only at infinity, and the corresponding eigenfunctions (and higher order eigenfunctions) form a complete basis in $L^{2}$. Similar results are proved in the current article for a periodic domain and mild solutions in $H^{\ell}(\ell=0,1,2, \cdots)$. The proof involves some deep result from Hilbert-Schmidt operators. Together with some deep result from sectorial operator, we prove the existence of invariant manifolds.

In terms of well-posedness of Navier-Stokes equations (NS), zero-viscosity limit problem has been extensively studied $[\mathbf{7}][\mathbf{2 6}]$. Here we are also interested in the zero-viscosity limit $\left(\nu \rightarrow 0^{+}\right)$problem for invariant manifolds. It is proved in this article that under an assumption, their sizes are of order $O(\sqrt{\nu})$ or $O(\nu)$ as $\nu \rightarrow 0^{+}$. Existence of invariant manifolds for Euler equations is still open. I tend to believe that invariant manifolds for Euler equations do not exist, at least not in the usual sense. An intuitive argument is as follows: Kato's technique [7] for proving the existence of a mild solution to Euler equations amounts to writing the solution as $u(t)=e^{-\int_{0}^{t} u(\tau) \cdot \nabla d \tau} u(0)$, where $u$ is the velocity, and the Leray projection and the external force are not included, which do not affect the argument. Notice that the $u(\tau)$ in the exponent depends upon $u(0)$, therefore, one should only expect the evolution operator of Euler equations to be at best $C^{0}$ in $u(0)$, just as in $t$. On the other hand, the existence of an invariant manifold is really an at least $C^{1}$ in $u(0)$ 
phenomenon. One can obtain expressions of invariant manifolds around a shear for a Galerkin truncation of the Euler equation [10], which exhibit a lip structure.

For concrete examples, e.g. single mode steady states, a continued fraction technique can be designed for calculating the eigenvalues [16]. Detailed calculations were conducted by Vincent Liu [13] [14]. Liu obtained rather detailed information on unstable eigenvalues. In some case, Liu showed that as $\nu \rightarrow 0^{+}$, the unstable eigenvalue does converge to that of linear Euler. In this article, besides the example of Liu, we will study two more examples. For the first example, a complete eigenvalue information is obtained, and the existence of unstable and stable manifolds is proved without any assumption. For the second example, only partial information on the eigenvalues is obtained, as in the example of Liu.

The article is organized as follows: In section 2, we will present the formulation of the problems. Section 3 is on invariant manifolds. Examples are studied in Section 4 .

\section{Formulation of the Problems}

We will study the following form of 2D Navier-Stokes equation (NS)

$$
\partial_{t} \Omega+\{\Psi, \Omega\}=\nu[\Delta \Omega+f(x)],
$$

where $\Omega$ is the vorticity which is a real scalar-valued function of three variables $t$ and $x=\left(x_{1}, x_{2}\right)$, the bracket $\{$,$\} is defined as$

$$
\{f, g\}=\left(\partial_{x_{1}} f\right)\left(\partial_{x_{2}} g\right)-\left(\partial_{x_{2}} f\right)\left(\partial_{x_{1}} g\right) \text {, }
$$

where $\Psi$ is the stream function given by,

$$
u_{1}=-\partial_{x_{2}} \Psi, \quad u_{2}=\partial_{x_{1}} \Psi,
$$

the relation between vorticity $\Omega$ and stream function $\Psi$ is,

$$
\Omega=\partial_{x_{1}} u_{2}-\partial_{x_{2}} u_{1}=\Delta \Psi,
$$

and $\nu$ is the viscosity, $\Delta$ is the $2 \mathrm{D}$ Laplacian, and $f(x)$ is the body force. We will pose periodic boundary condition

$$
\Omega\left(t, x_{1}+2 \pi, x_{2}\right)=\Omega\left(t, x_{1}, x_{2}\right)=\Omega\left(t, x_{1}, x_{2}+2 \pi\right),
$$

i.e. the $2 \mathrm{D}$ NS is defined on the 2 -torus $\mathbb{T}^{2}$. We require that $\Psi$ and $f$ have mean zero

$$
\int_{\mathbb{T}^{2}} \Psi d x=\int_{\mathbb{T}^{2}} f d x=0
$$

Of course $\Omega$ always has mean zero. In this case, $\Psi=\Delta^{-1} \Omega$.

Setting $\nu=0$ in the 2D NS (2.1), one gets the corresponding 2D Euler equation. We will study the common steady states of 2D NS and 2D Euler given by

$$
\{\Psi, \Omega\}=0, \quad \Delta \Omega+f(x)=0 .
$$

Our three prime examples are: (1). The shear $\Omega=\cos x_{2}$ defined on the rectangular periodic domain $[0,2 \pi / \alpha] \times[0,2 \pi]$ where $1 / 2<\alpha<1$. (2). The shear $\Omega=$ $\cos \left(x_{1}+x_{2}\right)$ where $f(x)=2 \cos \left(x_{1}+x_{2}\right)$. (3). The cat's eye $\Omega=\cos x_{1}+\alpha \cos x_{2}$ where $\alpha$ is a constant and $f(x)=\cos x_{1}+\alpha \cos x_{2}$.

We will study the following form of 3D Navier-Stokes equation

$$
\partial_{t} \Omega+(u \cdot \nabla) \Omega-(\Omega \cdot \nabla) u=\nu[\Delta \Omega+f(x)],
$$


where $u=\left(u_{1}, u_{2}, u_{3}\right)$ is the velocity, $\Omega=\left(\Omega_{1}, \Omega_{2}, \Omega_{3}\right)$ is the vorticity, $\nabla=$ $\left(\partial_{x_{1}}, \partial_{x_{2}}, \partial_{x_{3}}\right), \Omega=\nabla \times u, \nabla \cdot u=0, \nu$ is the viscosity, $\Delta$ is the 3D Laplacian, and $f(x)=\left(f\left(x_{1}\right), f\left(x_{2}\right), f\left(x_{3}\right)\right)$ is the body force. We also pose periodic boundary condition of period $(2 \pi, 2 \pi, 2 \pi)$, i.e. the $3 \mathrm{D} \mathrm{NS}$ is defined on the 3 -torus $\mathbb{T}^{3}$. We require that $u, f$ and $\Omega$ all have mean zero. In this case, $u$ can be uniquely determined from $\Omega$ by Fourier transform:

$$
\begin{aligned}
& \tilde{u}_{1}(k)=i|k|^{-2}\left[k_{2} \tilde{\Omega}_{3}(k)-k_{3} \tilde{\Omega}_{2}(k)\right], \\
& \tilde{u}_{2}(k)=i|k|^{-2}\left[k_{3} \tilde{\Omega}_{1}(k)-k_{1} \tilde{\Omega}_{3}(k)\right], \\
& \tilde{u}_{3}(k)=i|k|^{-2}\left[k_{1} \tilde{\Omega}_{2}(k)-k_{2} \tilde{\Omega}_{1}(k)\right] .
\end{aligned}
$$

Setting $\nu=0$ in the 3D NS (2.3), one gets the corresponding 3D Euler equation. We will study the common steady states of 3D NS and 3D Euler given by

$$
(u \cdot \nabla) \Omega-(\Omega \cdot \nabla) u=0, \quad \Delta \Omega+f(x)=0 .
$$

Our prime example is: The ABC flow

$u_{1}=A \sin x_{3}+C \cos x_{2}, \quad u_{2}=B \sin x_{1}+A \cos x_{3}, \quad u_{3}=C \sin x_{2}+B \cos x_{1}$.

Here $\Omega=u=f$.

\section{Invariant Manifolds}

Centering around a $C^{\infty}$ steady state $\Omega_{*}, u_{*}, \Psi_{*}$ given by (2.2) or (2.4), one can rewrite the NS as

$$
\partial_{t} \Omega=L \Omega+N(\Omega)
$$

where for $2 \mathrm{D}$

and for $3 \mathrm{D}$

$$
\begin{aligned}
L \Omega & =\nu \Delta \Omega-\left\{\Psi_{*}, \Omega\right\}-\left\{\Psi, \Omega_{*}\right\}, \\
N(\Omega) & =-\{\Psi, \Omega\}
\end{aligned}
$$

$$
\begin{aligned}
L \Omega & =\nu \Delta \Omega-\left(u_{*} \cdot \nabla\right) \Omega-(u \cdot \nabla) \Omega_{*} \\
& +\left(\Omega_{*} \cdot \nabla\right) u+(\Omega \cdot \nabla) u_{*}, \\
N(\Omega) & =-(u \cdot \nabla) \Omega+(\Omega \cdot \nabla) u .
\end{aligned}
$$

THEOREM 3.1. The linear Euler operator $L$ is a closed operator. Its spectrum consists of infinitely many discrete eigenvalues, each of finite multiplicity, which can cluster only at infinity. All the eigenvalues lie inside the parabolic region

$$
\lambda_{r} \leq a-b \lambda_{i}^{2}
$$

where $a>0$ and $b>0$ are some constants, and $\lambda=\lambda_{r}+i \lambda_{i}$ is the spectral parameter. The corresponding eigenfunctions (and higher order eigenfunctions) form a complete basis in $H^{\ell}(\ell=0,1,2, \cdots)$.

Proof. The proof for $L$ being a closed operator is trivial. We can write $L$ as

$$
L=\nu \Delta+A
$$

where $A$ is relatively compact with respect to $\nu \Delta$. By Weyl's essential spectrum theorem [23], $L$ and $\nu \Delta$ have the same essential spectrum. Since the essential spectrum of $\nu \Delta$ is empty, so is $L$. Therefore, $L$ has only eigenvalues. It is easy to see that for large enough $\lambda>0,(L-\lambda)^{-1}$ is compact, thus each eigenvalue can be of only finite multiplicity and all the eigenvalues can cluster only at infinity $[\mathbf{2}]$. 
Next we show that large enough $\lambda>0$ is not an eigenvalue. Assume the contrary, then there exists nonzero $\Omega \in H^{\ell+2}$ such that

$$
\lambda \Omega-\nu \Delta \Omega=A \Omega .
$$

Applying $(-\Delta)^{\ell / 2}$, one gets

$$
\lambda(-\Delta)^{\ell / 2} \Omega-\nu \Delta(-\Delta)^{\ell / 2} \Omega=(-\Delta)^{\ell / 2}(A \Omega) .
$$

Multiplying by $(-\Delta)^{\ell / 2} \Omega$, one obtains

$$
\begin{aligned}
& \lambda\left|(-\Delta)^{\ell / 2} \Omega\right|^{2}+\nu\left[(-\Delta)^{\ell / 2} \Omega\right] \cdot\left[(-\Delta)(-\Delta)^{\ell / 2} \Omega\right] \\
= & {\left[(-\Delta)^{\ell / 2} \Omega\right] \cdot\left[(-\Delta)^{\ell / 2}(A \Omega)\right] . }
\end{aligned}
$$

Thus

$$
\begin{aligned}
& \lambda \int\left|(-\Delta)^{\ell / 2} \Omega\right|^{2}+\nu \int\left|(-\Delta)^{(\ell+1) / 2} \Omega\right|^{2} \\
= & \int\left[(-\Delta)^{\ell / 2} \Omega\right] \cdot\left[(-\Delta)^{\ell / 2}(A \Omega)\right] \\
\leq & C \int\left|(-\Delta)^{\ell / 2} \Omega\right|\left|(-\Delta)^{(\ell+1) / 2} \Omega\right| \\
\leq & C\left(\epsilon^{2} \int\left|(-\Delta)^{(\ell+1) / 2} \Omega\right|^{2}+\frac{1}{\epsilon^{2}} \int\left|(-\Delta)^{\ell / 2} \Omega\right|^{2}\right) .
\end{aligned}
$$

Let $\epsilon^{2}=\frac{\nu}{2 C}$ and $\lambda=\frac{2 C^{2}}{\nu}+1$, we have

$$
\int\left|(-\Delta)^{\ell / 2} \Omega\right|^{2}+\frac{1}{2} \nu \int\left|(-\Delta)^{(\ell+1) / 2} \Omega\right|^{2} \leq 0,
$$

which is a contradiction to $\Omega$ being an eigenfunction. Thus $\lambda=\frac{2 C^{2}}{\nu}+1$ is in the resolvent set. Next let $\lambda$ be a complex number in the resolvent set. Naturally, we need to consider $\Omega$ as a complex-valued function. For any $h \in H^{\ell}$, there is a $\Omega \in H^{\ell+2}$ such that

$$
-\nu \Delta \Omega+\lambda \Omega-A \Omega=h
$$

Applying $(-\Delta)^{\ell / 2}$, one gets

$$
-\nu \Delta(-\Delta)^{\ell / 2} \Omega+\lambda(-\Delta)^{\ell / 2} \Omega-(-\Delta)^{\ell / 2}(A \Omega)=(-\Delta)^{\ell / 2} h .
$$

Multiplying by $(-\Delta)^{\ell / 2} \bar{\Omega}$, one obtains

$$
\begin{aligned}
& \nu\left[(-\Delta)(-\Delta)^{\ell / 2} \Omega\right] \cdot\left[(-\Delta)^{\ell / 2} \bar{\Omega}\right]+\lambda\left[(-\Delta)^{\ell / 2} \Omega\right] \cdot\left[(-\Delta)^{\ell / 2} \bar{\Omega}\right] \\
= & {\left[(-\Delta)^{\ell / 2}(A \Omega)\right] \cdot\left[(-\Delta)^{\ell / 2} \bar{\Omega}\right]+\left[(-\Delta)^{\ell / 2} h\right] \cdot\left[(-\Delta)^{\ell / 2} \bar{\Omega}\right] . }
\end{aligned}
$$

Thus

$$
\begin{aligned}
& \nu \int\left|(-\Delta)^{(\ell+1) / 2} \Omega\right|^{2}+\lambda \int\left|(-\Delta)^{\ell / 2} \Omega\right|^{2} \\
= & \int\left[(-\Delta)^{\ell / 2}(A \Omega)\right] \cdot\left[(-\Delta)^{\ell / 2} \bar{\Omega}\right]+\int\left[(-\Delta)^{\ell / 2} h\right] \cdot\left[(-\Delta)^{\ell / 2} \bar{\Omega}\right] .
\end{aligned}
$$


Taking the real part, one gets

$$
\begin{aligned}
\nu\|\Omega\|_{\ell+1}^{2}+\lambda_{r}\|\Omega\|_{\ell}^{2} & \leq C\left(\epsilon^{2}\|\Omega\|_{\ell+1}^{2}+\frac{1}{\epsilon^{2}}\|\Omega\|_{\ell}^{2}\right) \\
& +\frac{1}{2}\left(\|h\|_{\ell}^{2}+\|\Omega\|_{\ell}^{2}\right) .
\end{aligned}
$$

Let $\epsilon^{2}=\frac{\nu}{2 C}$, one has

$$
\frac{1}{2} \nu\|\Omega\|_{\ell+1}^{2}+\lambda_{r}\|\Omega\|_{\ell}^{2} \leq \frac{1}{2}\|h\|_{\ell}^{2}+C_{1}\|\Omega\|_{\ell}^{2} .
$$

Taking the imaginary part of (3.2), one gets

$$
\lambda_{i}\|\Omega\|_{\ell}^{2} \leq C_{2}\|\Omega\|_{\ell+1}\|\Omega\|_{\ell}+\|h\|_{\ell}\|\Omega\|_{\ell},
$$

that is

$$
\lambda_{i}\|\Omega\|_{\ell} \leq C_{2}\|\Omega\|_{\ell+1}+\|h\|_{\ell} .
$$

Then we have

$$
\lambda_{i}^{2}\|\Omega\|_{\ell}^{2} \leq C_{3}\|\Omega\|_{\ell+1}^{2}+C_{4}\|h\|_{\ell}^{2},
$$

that is

$$
\frac{\nu}{2 C_{3}} \lambda_{i}^{2}\|\Omega\|_{\ell}^{2} \leq \frac{1}{2} \nu\|\Omega\|_{\ell+1}^{2}+\frac{\nu C_{4}}{2 C_{3}}\|h\|_{\ell}^{2} .
$$

Adding (3.3) and (3.4), we have

$$
\left(\lambda_{r}+\frac{\nu}{2 C_{3}} \lambda_{i}^{2}-C_{1}\right)\|\Omega\|_{\ell}^{2} \leq \frac{1}{2}\left(1+\frac{\nu C_{4}}{C_{3}}\right)\|h\|_{\ell}^{2} .
$$

Let $a=C_{1}, b=\frac{\nu}{2 C_{3}}$, and $C_{5}=\frac{1}{2}\left(1+\frac{\nu C_{4}}{C_{3}}\right)$,

$$
\left(\lambda_{r}-a+b \lambda_{i}^{2}\right)\|\Omega\|_{\ell}^{2} \leq C_{5}\|h\|_{\ell}^{2} .
$$

Thus if $\lambda_{r}-a+b \lambda_{i}^{2}>0$,

$$
\left\|(L-\lambda)^{-1}\right\| \leq \frac{C_{5}^{1 / 2}}{\left(\lambda_{r}-a+b \lambda_{i}^{2}\right)^{1 / 2}} .
$$

$(L-\lambda)^{-1}$ is holomorphic in $\lambda$ and can be continued analytically as long as $\|(L-$ $\lambda)^{-1} \|$ is bounded. As shown above, $(L-\lambda)^{-1}$ exists at large $\lambda>0$. Then from (3.5), $(L-\lambda)^{-1}$ exists everywhere outside the parabolic region $\lambda_{r} \leq a-b \lambda_{i}^{2}$. Thus the eigenvalues of $L$ lie inside this region. To prove the rest of the theorem, we need a known lemma [Corollary 31, pp.1042, [3]].

Lemma 3.2. Let $T$ be a densely defined unbounded linear operator in a Hilbert space $H$, with the property that for some $\lambda_{0}$ in the resolvent set of $T,\left(T-\lambda_{0}\right)^{-1}$ is a Hilbert-Schmidt operator. Let $\gamma_{1}, \cdots, \gamma_{5}$ be non-overlapping differentiable arcs having limiting directions at infinity, and such that no adjacent pair of arcs form an angle as great as $\pi / 2$ at infinity. Suppose that the resolvent $(T-\lambda)^{-1}$ satisfies an inequality $\left\|(T-\lambda)^{-1}\right\|=O\left(|\lambda|^{-N}\right)$ as $\lambda \rightarrow \infty$ along each arc $\gamma_{j}$ for some natural number $N$. Then the eigenfunctions (and higher order eigenfunctions) form a complete basis in $H$. 
From (3.5), we see that along any ray $\lambda_{r}=c \lambda_{i}$ ( $c$ finite),

$$
\left\|(L-\lambda)^{-1}\right\|=O\left(|\lambda|^{-1}\right) \quad \text { as } \quad \lambda \rightarrow \infty .
$$

Next we show that $(L-\lambda)^{-1}$ is a Hilbert-Schmidt operator for large enough $\lambda>0$. Notice that

$$
(L-\lambda)^{-1}=\left[I+(\nu \Delta-\lambda)^{-1} A\right]^{-1}(\nu \Delta-\lambda)^{-1} .
$$

Since

$$
\begin{aligned}
& \left\|(\nu \Delta-\lambda)^{-1} \Omega\right\|_{\ell}^{2}=\sum_{k} \frac{|k|^{2 \ell}}{\left(\nu|k|^{2}+\lambda\right)^{2}}\left|\Omega_{k}\right|^{2} \\
& =\frac{1}{\nu^{2}} \sum_{k} \frac{1}{|k|^{2}+\lambda / \nu} \frac{|k|^{2}}{|k|^{2}+\lambda / \nu}|k|^{2(\ell-1)}\left|\Omega_{k}\right|^{2} \\
& \leq \frac{1}{\nu \lambda} \sum_{k}|k|^{2(\ell-1)}\left|\Omega_{k}\right|^{2}
\end{aligned}
$$

we have

$$
\left\|(\nu \Delta-\lambda)^{-1} \Omega\right\|_{\ell} \leq \frac{1}{\sqrt{\nu \lambda}}\|\Omega\|_{\ell-1}
$$

It is easy to see that

$$
\|A \Omega\|_{\ell-1} \leq C\|\Omega\|_{\ell} .
$$

Thus

$$
\left\|(\nu \Delta-\lambda)^{-1} A \Omega\right\|_{\ell} \leq \frac{C}{\sqrt{\nu \lambda}}\|\Omega\|_{\ell} .
$$

Choose $\lambda_{0}=\frac{2 C^{2}}{\nu}$, then

$$
\left\|\left(\nu \Delta-\lambda_{0}\right)^{-1} A\right\| \leq \frac{1}{2} .
$$

We have $\left[I+\left(\nu \Delta-\lambda_{0}\right)^{-1} A\right]^{-1}$ being a bounded operator. Recall that a bounded linear operator $T$ is a Hilbert-Schmidt operator if

$$
\sum\left\|T e_{k}\right\|^{2}
$$

is finite where $\left\{e_{k}\right\}$ is a complete orthonormal basis [3]. By using the Fourier basis for $H^{\ell}$, one sees that $\left(\nu \Delta-\lambda_{0}\right)^{-1}$ is a Hilbert-Schmidt operator. A product of a bounded linear operator with a Hilbert-Schmidt operator is another HilbertSchmidt operator. Thus $\left(L-\lambda_{0}\right)^{-1}$ is a Hilbert-Schmidt operator. By Lemma 3.2 , the corresponding eigenfunctions (and higher order eigenfunctions) of $L$ form a complete basis in $H^{\ell}(\ell=0,1,2, \cdots)$.

We will study the case as described by the following set-up.

- Set-Up. Let there be $m$ unstable eigenvalues $\lambda_{1}^{u} \cdots \lambda_{m}^{u}, n$ center eigenvalues $\lambda_{1}^{c} \cdots \lambda_{n}^{c}$, and of course the rest infinitely many stable eigenvalues $\lambda_{1}^{s} \lambda_{2}^{s} \cdots$. Let the real parts of $\lambda_{1}^{u}$ and $\lambda_{1}^{s}$ have the smallest absolute values among the unstable and stable eigenvalues, respectively.

REMARK 3.3. For specific examples, often there is no center eigenvalue, here we put them in for generality. We need $H^{\ell}(\ell=0,1,2, \cdots)$ to be a Banach algebra [1]. Thus for $2 \mathrm{D}$ and $3 \mathrm{D}, \ell \geq 2$. Our argument requires that we work with $H^{\ell}$ where $\ell \geq 3$ for both $2 \mathrm{D}$ and $3 \mathrm{D}$. 
We have the splitting

$$
H^{\ell}=H_{u}^{\ell}+H_{c}^{\ell}+H_{s}^{\ell}
$$

where $H_{z}^{\ell}(z=u, c, s)$ are spanned by the corresponding eigenfunctions. Projections onto $H_{z}^{\ell}$ are denoted by $P^{z}(z=u, c, s)$. Denote by

$$
L^{z}=P^{z} L, \quad N^{z}=P^{z} N, \quad \Omega^{z}=P^{z} \Omega, \quad(z=u, c, s) .
$$

Then (3.1) can be rewritten as

$$
\partial_{t} \Omega^{z}=L^{z} \Omega^{z}+N^{z}(\Omega), \quad(z=u, c, s) .
$$

Since in this case the spectral mapping theorem is trivially true, one has the trichotomy:

$$
\begin{aligned}
& \left\|e^{t L^{u}}\right\| \leq C e^{\alpha t}, \quad t \leq 0 ; \\
& \left\|e^{t L^{c}}\right\| \leq C|t|^{n_{1}}, \quad t \in \mathbb{R} ; \\
& \left\|e^{t L^{s}}\right\| \leq C e^{-\beta t}, \quad t \geq 0 ;
\end{aligned}
$$

where $\alpha=\operatorname{Re}\left\{\lambda_{1}^{u}\right\}-\epsilon, \beta=-\operatorname{Re}\left\{\lambda_{1}^{s}\right\}-\epsilon, n_{1} \leq n$, and $0<\epsilon \ll-\operatorname{Re}\left\{\lambda_{1}^{s}\right\}$. Our argument depends upon the following two crucial facts:

(1) One has a stronger inequality than (3.9) [[5], Theorem 1.5.4] due to the fact that $L^{s}$ is sectorial,

$$
\left\|e^{t L^{s}} \Omega^{s}\right\|_{\ell+1} \leq C(\beta t)^{-1 / 2} e^{-\beta t}\left\|\Omega^{s}\right\|_{\ell}, \quad t \geq 0 .
$$

(2) Due to finite dimensionality, one has

$$
\left\|\Omega^{z}\right\|_{\ell+1} \leq C\left\|\Omega^{z}\right\|_{\ell}, \quad(z=u, c) .
$$

REMARK 3.4. For (3.11), take $z=u$ for example, since $\Omega_{1}^{u} \cdots \Omega_{m}^{u}$ belong to $H^{\ell}$ for any $\ell$, we can take

$$
C=\max _{1 \leq j \leq m}\left\{\left\|\Omega_{j}^{u}\right\|_{\ell+1} /\left\|\Omega_{j}^{u}\right\|_{\ell}\right\} .
$$

TheOREM 3.5. In a neighborhood of the fixed point $\Omega_{*}$ in the Sobolev space $H^{\ell}\left(\mathbb{T}^{d}\right)(\ell \geq 3, d=2,3)$, there exist a $m$-dimensional $C^{\infty}$ unstable manifold, a $n$-dimensional $C^{\infty}$ center manifold, a $(m+n)$-codimensional $C^{\infty}$ stable manifold, $a(m+n)$-dimensional $C^{\infty}$ center-unstable manifold, and a $m$-codimensional $C^{\infty}$ center-stable manifold.

Proof. We will take the center-stable manifold as the example. The arguments for the others are similar. Apply the method of variation of parameters to (3.6), one gets the integral equations

$$
\Omega^{z}(t)=e^{\left(t-t_{0}\right) L^{z}} \Omega^{z}\left(t_{0}\right)+\int_{t_{0}}^{t} e^{(t-\tau) L^{z}} N^{z}(\Omega) d \tau, \quad(z=u, c, s) .
$$

To prove the existence of a center-stable manifold, we start with the Banach space

$$
B_{\gamma, \ell}=\left\{\Omega(t) \mid\|\Omega\|_{\gamma, \ell}=\sup _{t \geq 0} e^{-\gamma t} \sum_{z=u, c, s}\left\|\Omega^{z}(t)\right\|_{\ell}<+\infty, \quad\left(\frac{1}{4} \alpha<\gamma<\alpha\right)\right\} .
$$

For such $\Omega(t)$ in $B_{\gamma, \ell}$

$$
\lim _{t_{0} \rightarrow+\infty} e^{\left(t-t_{0}\right) L^{u}} \Omega^{u}\left(t_{0}\right) \rightarrow 0
$$


In the equation (3.13), for $z=u$, let $t_{0} \rightarrow+\infty$; for $z=c$, s, let $t_{0}=0$; then one gets

$$
\begin{aligned}
& \Omega^{u}(t)=\int_{+\infty}^{t} e^{(t-\tau) L^{u}} N^{u}(\Omega) d \tau, \\
& \Omega^{z}(t)=e^{t L^{z}} \Omega^{z}(0)+\int_{0}^{t} e^{(t-\tau) L^{z}} N^{z}(\Omega) d \tau, \quad(z=c, s) .
\end{aligned}
$$

The right hand side of (3.14)-(3.15) defines a map $\Gamma=\Gamma^{u}+\Gamma^{c}+\Gamma^{s}$ on $B_{\gamma, \ell}$. First we show that $\Gamma$ contracts, then $\Gamma$ acting is an easier argument. From (3.14), one has

$$
e^{-\gamma t}\left\|\Gamma^{u} \Omega_{1}-\Gamma^{u} \Omega_{2}\right\|_{\ell} \leq C \int_{t}^{+\infty} e^{-\gamma t} e^{\alpha(t-\tau)}\left\|N^{u}\left(\Omega_{1}\right)-N^{u}\left(\Omega_{2}\right)\right\|_{\ell} d \tau .
$$

Using (3.11), one gets

$$
e^{-\gamma t}\left\|\Gamma^{u} \Omega_{1}-\Gamma^{u} \Omega_{2}\right\|_{\ell} \leq C \int_{t}^{+\infty} e^{-\gamma t} e^{\alpha(t-\tau)}\left\|N^{u}\left(\Omega_{1}\right)-N^{u}\left(\Omega_{2}\right)\right\|_{\ell-1} d \tau,
$$

in this article, all constants are denoted by the same $C$. Since $H^{\ell-1}$ is a Banach algebra

$$
\left\|N^{u}\left(\Omega_{1}\right)-N^{u}\left(\Omega_{2}\right)\right\|_{\ell-1} \leq C\left(\left\|\Omega_{1}\right\|_{\ell}+\left\|\Omega_{2}\right\|_{\ell}\right)\left\|\Omega_{1}-\Omega_{2}\right\|_{\ell} .
$$

Finally one gets

$$
e^{-\gamma t}\left\|\Gamma^{u} \Omega_{1}-\Gamma^{u} \Omega_{2}\right\|_{\ell} \leq C \int_{t}^{+\infty} e^{(\alpha-\gamma)(t-\tau)}\left(\left\|\Omega_{1}\right\|_{\ell}+\left\|\Omega_{2}\right\|_{\ell}\right) e^{-\gamma \tau}\left\|\Omega_{1}-\Omega_{2}\right\|_{\ell} d \tau .
$$

Now we need to replace $N(\Omega)$ by its cut-off $\chi\left(\|\Omega\|_{\ell} / \delta\right) N(\Omega)$ for some $\delta>0$, where

$$
\begin{aligned}
& \chi(r)=1, \quad r \in[0,1] ; \quad \chi(r)=0, \quad r \in[3,+\infty) ; \\
& \chi^{\prime}(r)<1, \quad r \in[0,+\infty) ; \quad \chi \in C_{0}^{\infty}(\mathbb{R}, \mathbb{R}) .
\end{aligned}
$$

If both $\chi\left(\left\|\Omega_{1}\right\|_{\ell} / \delta\right)$ and $\chi\left(\left\|\Omega_{2}\right\|_{\ell} / \delta\right)$ are zero, then of course

$$
\chi\left(\left\|\Omega_{1}\right\|_{\ell} / \delta\right) N\left(\Omega_{1}\right)-\chi\left(\left\|\Omega_{2}\right\|_{\ell} / \delta\right) N\left(\Omega_{2}\right)=0 .
$$

If one of them is nonzero, without loss of generality, say $\chi\left(\left\|\Omega_{1}\right\|_{\ell} / \delta\right) \neq 0$, then

$$
\begin{aligned}
& \left\|\chi\left(\left\|\Omega_{1}\right\|_{\ell} / \delta\right) N\left(\Omega_{1}\right)-\chi\left(\left\|\Omega_{2}\right\|_{\ell} / \delta\right) N\left(\Omega_{2}\right)\right\|_{\ell-1} \\
\leq & \left\|\left[\chi\left(\left\|\Omega_{1}\right\|_{\ell} / \delta\right)-\chi\left(\left\|\Omega_{2}\right\|_{\ell} / \delta\right)\right] N\left(\Omega_{1}\right)\right\|_{\ell-1}+\left\|\chi\left(\left\|\Omega_{2}\right\|_{\ell} / \delta\right)\left[N\left(\Omega_{1}\right)-N\left(\Omega_{2}\right)\right]\right\|_{\ell-1} \\
\leq & \left|\chi\left(\left\|\Omega_{1}\right\|_{\ell} / \delta\right)-\chi\left(\left\|\Omega_{2}\right\|_{\ell} / \delta\right)\right|\left\|N\left(\Omega_{1}\right)\right\|_{\ell-1}+\left|\chi\left(\left\|\Omega_{2}\right\|_{\ell} / \delta\right)\right|\left\|N\left(\Omega_{1}\right)-N\left(\Omega_{2}\right)\right\|_{\ell-1} \\
\leq & \frac{1}{\delta}\left\|\Omega_{1}-\Omega_{2}\right\|_{\ell}\left\|N\left(\Omega_{1}\right)\right\|_{\ell-1}+\left|\chi\left(\left\|\Omega_{2}\right\|_{\ell} / \delta\right)\right|\left\|N\left(\Omega_{1}\right)-N\left(\Omega_{2}\right)\right\|_{\ell-1} .
\end{aligned}
$$

If $\chi\left(\left\|\Omega_{2}\right\|_{\ell} / \delta\right)=0$, then the second part disappears; otherwise, $\left\|\Omega_{2}\right\|_{\ell}<3 \delta$. In any case, one gets

$$
\left\|\chi\left(\left\|\Omega_{1}\right\|_{\ell} / \delta\right) N\left(\Omega_{1}\right)-\chi\left(\left\|\Omega_{2}\right\|_{\ell} / \delta\right) N\left(\Omega_{2}\right)\right\|_{\ell-1} \leq C \delta\left\|\Omega_{1}-\Omega_{2}\right\|_{\ell} .
$$

Thus, one has

$$
\begin{aligned}
e^{-\gamma t}\left\|\Gamma^{u} \Omega_{1}-\Gamma^{u} \Omega_{2}\right\|_{\ell} & \leq C \delta\left\|\Omega_{1}-\Omega_{2}\right\|_{\gamma, \ell} \int_{t}^{+\infty} e^{(\alpha-\gamma)(t-\tau)} d \tau \\
& =C \frac{\delta}{\alpha-\gamma}\left\|\Omega_{1}-\Omega_{2}\right\|_{\gamma, \ell} .
\end{aligned}
$$


Thus

$$
\sup _{t \geq 0} e^{-\gamma t}\left\|\Gamma^{u} \Omega_{1}-\Gamma^{u} \Omega_{2}\right\|_{\ell} \leq C \frac{\delta}{\alpha-\gamma}\left\|\Omega_{1}-\Omega_{2}\right\|_{\gamma, \ell} .
$$

For $z=c$ in (3.15), one has

$$
e^{-\gamma t}\left\|\Gamma^{c} \Omega_{1}-\Gamma^{c} \Omega_{2}\right\|_{\ell} \leq C \int_{0}^{t} e^{-\gamma t} e^{\epsilon(t-\tau)}\left\|N^{c}\left(\Omega_{1}\right)-N^{c}\left(\Omega_{2}\right)\right\|_{\ell} d \tau
$$

here notice that $\Omega_{1}^{c}$ and $\Omega_{2}^{c}$ have the same initial condition $\Omega_{1}^{c}(0)=\Omega_{2}^{c}(0)$. The same argument as above leads to

$$
\begin{aligned}
e^{-\gamma t}\left\|\Gamma^{c} \Omega_{1}-\Gamma^{c} \Omega_{2}\right\|_{\ell} & \leq C \delta\left\|\Omega_{1}-\Omega_{2}\right\|_{\gamma, \ell} \int_{0}^{t} e^{-(\gamma-\epsilon)(t-\tau)} d \tau \\
& \leq C \frac{\delta}{\gamma-\epsilon}\left\|\Omega_{1}-\Omega_{2}\right\|_{\gamma, \ell} .
\end{aligned}
$$

Thus

$$
\sup _{t \geq 0} e^{-\gamma t}\left\|\Gamma^{c} \Omega_{1}-\Gamma^{c} \Omega_{2}\right\|_{\ell} \leq C \frac{\delta}{\gamma-\epsilon}\left\|\Omega_{1}-\Omega_{2}\right\|_{\gamma, \ell} .
$$

For $z=s$ in (3.15), one has

$$
e^{-\gamma t}\left\|\Gamma^{s} \Omega_{1}-\Gamma^{s} \Omega_{2}\right\|_{\ell} \leq C \int_{0}^{t} e^{-\gamma t}[\beta(t-\tau)]^{-1 / 2} e^{-\beta(t-\tau)}\left\|N^{s}\left(\Omega_{1}\right)-N^{s}\left(\Omega_{2}\right)\right\|_{\ell-1} d \tau .
$$

The same argument as before leads to

$$
\begin{aligned}
e^{-\gamma t}\left\|\Gamma^{s} \Omega_{1}-\Gamma^{s} \Omega_{2}\right\|_{\ell} & \leq C \delta\left\|\Omega_{1}-\Omega_{2}\right\|_{\gamma, \ell} \int_{0}^{t}[\beta(t-\tau)]^{-1 / 2} e^{-(\beta+\gamma)(t-\tau)} d \tau \\
& \leq C \frac{\delta}{\sqrt{\beta}}\left\|\Omega_{1}-\Omega_{2}\right\|_{\gamma, \ell} .
\end{aligned}
$$

Thus

$$
\sup _{t \geq 0} e^{-\gamma t}\left\|\Gamma^{s} \Omega_{1}-\Gamma^{s} \Omega_{2}\right\|_{\ell} \leq C \frac{\delta}{\sqrt{\beta}}\left\|\Omega_{1}-\Omega_{2}\right\|_{\gamma, \ell}
$$

By choosing

$$
\delta=\min \left\{\frac{1}{6} C(\alpha-\gamma), \frac{1}{6} C(\gamma-\epsilon), \frac{1}{6} C \sqrt{\beta}\right\},
$$

(3.17)-(3.19) imply that

$$
\left\|\Gamma \Omega_{1}-\Gamma \Omega_{2}\right\|_{\gamma, \ell} \leq \frac{1}{2}\left\|\Omega_{1}-\Omega_{2}\right\|_{\gamma, \ell} .
$$

Thus $\Gamma$ contracts. The claim that $\Gamma$ acts, i.e. $\Gamma: B_{\gamma, \ell} \rightarrow B_{\gamma, \ell} ;$ follows similarly, and is an easier argument. By the contraction mapping theorem, $\Gamma$ has a fixed point $\Omega$ in $B_{\gamma, \ell}$, which satisfies (3.14)-(3.15), of course, with $N$ replaced by its cut-off. Equations (3.14)-(3.15) define a map which maps $\Omega^{z}(0)(z=c, s)$ into

$$
\Omega^{u}(0)=\int_{+\infty}^{0} e^{-\tau L^{u}} N^{u}(\Omega) d \tau .
$$

This map defines the center-stable manifold. Due to the cut-off, only in the $\delta$ neighborhood of $\Omega_{*}$, this center-stable manifold corresponds to the NS flow. Smoothness can be proved through the standard argument $[\mathbf{1 1}]$. This proves the theorem. 
To study the zero-viscosity limits of invariant manifolds, we need to make an assumption.

- Assumption 1. As $\nu \rightarrow 0^{+}, \operatorname{Re}\left\{\lambda_{1}^{u}\right\}$ is of order $O(1)$, and $\operatorname{Re}\left\{\lambda_{1}^{s}\right\}$ is $O(\nu)$, and the constant $C$ in (3.7)-(3.11) is $O(1)$.

TheOREM 3.6. Under Assumption 1, as $\nu \rightarrow 0^{+}$, in an order $O(\sqrt{\nu})$ neighborhood of the fixed point $\Omega_{*}$ in the Sobolev space $H^{\ell}\left(\mathbb{T}^{d}\right)(\ell \geq 3, d=2,3)$, there exist an unstable manifold and a center-stable manifold; and in its order $O(\nu)$ neighborhood, there exist a stable manifold, a center manifold, and a center-unstable manifold.

Proof. Under the Assumption 1, the $\delta$ in (3.20) is of order $O(\sqrt{\nu})$ since $\beta$ is $O(\nu)$. Thus we have the claim for the center-stable manifold. By keeping track of similar estimates as in the proof of Theorem 3.5, one can quickly see the rest of the claims.

Due to Assumption 1, zero-viscosity limits of invariant manifolds become a challenging problem. The challenge is further amplified by the elusiveness of the invariant manifolds for Euler equations as discussed in the Introduction. As a test, we studied a simpler problem in [12], which reveals some unique features of the zero-viscosity limit of the spectra. For instance, the zero-viscosity limit of a discrete spectrum can be a continuous spectrum which is not the spectrum of zero-viscosity.

\section{Examples}

Using Fourier series for the 2D NS (2.1),

$$
\Omega=\sum_{k \in \mathbb{Z}^{2} /\{0\}} \omega_{k} e^{i k \cdot x}, \quad f=\sum_{k \in \mathbb{Z}^{2} /\{0\}} f_{k} e^{i k \cdot x},
$$

where $\omega_{-k}=\overline{\omega_{k}}$ and $f_{-k}=\overline{f_{k}}$, one gets the kinetic form of 2D NS

$$
\dot{\omega}_{k}=\sum_{k=q+r} A(q, r) \omega_{q} \omega_{r}+\nu\left[-|k|^{2} \omega_{k}+f_{k}\right],
$$

where

$$
A(q, r)=\frac{1}{2}\left[|r|^{-2}-|q|^{-2}\right]\left|\begin{array}{ll}
q_{1} & r_{1} \\
q_{2} & r_{2}
\end{array}\right|
$$

where $|q|^{2}=q_{1}^{2}+q_{2}^{2}$ for $q=\left(q_{1}, q_{2}\right)^{T}$, similarly for $r$. Linearize the 2D NS at the steady state given by a single mode,

$$
\Omega_{*}=\Gamma e^{i p \cdot x}+\bar{\Gamma} e^{-i p \cdot x}
$$

where $f=|p|^{2} \Omega_{*}$, one gets

$$
\begin{aligned}
\dot{\omega}_{\hat{k}+n p} & =A(p, \hat{k}+(n-1) p) \Gamma \omega_{\hat{k}+(n-1) p} \\
& +A(-p, \hat{k}+(n+1) p) \bar{\Gamma} \omega_{\hat{k}+(n+1) p}-\nu|\hat{k}+n p|^{2} \omega_{\hat{k}+n p},
\end{aligned}
$$

where its right hand side defines the linear NS operator $L$. Let $\omega_{\hat{k}+n p}=e^{\lambda t} \tilde{\omega}_{\hat{k}+n p}$, $\Gamma=|\Gamma| e^{i \gamma}, a=\frac{1}{2}|\Gamma|\left|\begin{array}{cc}p_{1} & \hat{k}_{1} \\ p_{2} & \hat{k}_{2}\end{array}\right|, \rho_{n}=|p|^{-2}-|\hat{k}+n p|^{-2}$, and $z_{n}=\rho_{n} e^{-i n \gamma} \tilde{\omega}_{\hat{k}+n p}$, then

$$
a_{n} z_{n}+z_{n-1}-z_{n+1}=0,
$$


where $a_{n}=\left(a \rho_{n}\right)^{-1}\left(\lambda+\nu|\hat{k}+n p|^{2}\right)$. Let $w_{n}=z_{n} / z_{n-1}$, one gets $[\mathbf{1 6}]$

$$
a_{n}+\frac{1}{w_{n}}=w_{n+1}
$$

which leads to the continued fraction,

$$
w_{n}^{(1)}=a_{n-1}+\frac{1}{a_{n-2}+\frac{1}{a_{n-3}+\cdots}} .
$$

Rewriting (4.4) as,

$$
w_{n}=\frac{1}{-a_{n}+w_{n+1}},
$$

which leads to the other continued fraction

$$
w_{n}^{(2)}=-\frac{1}{a_{n}+\frac{1}{a_{n+1}+\cdots}} .
$$

Along the spirit of the finite difference of a second order ordinary differential equation, the difference equation (4.3) should have two linearly independent solutions. Rigorous theory has been well developed. This is the so-called Poincaré-Perron system. For details, see [21] [18] [19] [20] [17] [4] [6] [15] [9] [14]. When $\nu>0$, $a_{n} \rightarrow \tilde{a} n^{2}$ as $|n| \rightarrow+\infty$. Then there are a growing solution $z_{n} / z_{n-1} \rightarrow \tilde{a} n^{2}$ and a decaying solution $z_{n} / z_{n-1} \rightarrow \tilde{a}^{-1} n^{-2}$, as $n \rightarrow+\infty$; and vice versa as $n \rightarrow-\infty$. The intuition on this is clear from (4.4) and (4.6). The eigenvalues are then determined by matching the two decaying solutions given by the two continued fractions (4.5) and (4.7). When $\nu=0, a_{n} \rightarrow \tilde{a}=a|p|^{2} \lambda$ as $|n| \rightarrow+\infty$. When $\operatorname{Re}\{\tilde{a}\} \neq 0$, or $\operatorname{Re}\{\tilde{a}\}=0(|\tilde{a}|>2)$, there are a growing solution $z_{n} / z_{n-1} \rightarrow w_{+},\left(\left|w_{+}\right|>1\right)$, and a decaying solution $z_{n} / z_{n-1} \rightarrow w_{-},\left(\left|w_{-}\right|<1\right)$, as $n \rightarrow+\infty$; and vice versa as $n \rightarrow-\infty$. Here $w_{ \pm}$solve the characteristic equation [9]

$$
w^{2}-\tilde{a} w-1=0 .
$$

Again the eigenvalues are determined by matching the two decaying solutions given by the two continued fractions (4.5) and (4.7). Finally, $\operatorname{Re}\{\tilde{a}\}=0(|\tilde{a}| \leq 2)$ corresponds to a continuous spectrum [9]. The proof of $[\mathbf{9}]$ can be generalized to $H^{\ell}$ setting for any natural number $\ell$. For more general result, see [25]. In summary, when $\nu>0$ or $\nu=0[\operatorname{Re}\{\tilde{a}\} \neq 0$, or $\operatorname{Re}\{\tilde{a}\}=0(|\tilde{a}|>2)]$, the eigenvalues are determined by

$$
a_{0}+\frac{1}{a_{-1}+\frac{1}{a_{-2}+\cdots}}=-\frac{1}{a_{1}+\frac{1}{a_{2}+\cdots}}
$$

which is obtained by matching $w_{n}^{(1)}(4.5)$ and $w_{n}^{(2)}(4.7)$ at $n=1$, i.e. $w_{1}^{(1)}=w_{1}^{(2)}$. The corresponding eigenfunctions belong to $H^{\ell}$ for any natural number $\ell$.

4.1. Example 1. Our first prime example is the shear $\Omega_{*}=\cos x_{2}$ defined on the rectangular periodic domain $[0,2 \pi / \alpha] \times[0,2 \pi]$ where $1 / 2<\alpha<1$. As shown below, this example has only one unstable eigenvalue. Complete information on this eigenvalue can be obtained. Thereby, existence of an unstable manifold and a stable manifold can be confirmed. This example is motivated by examples 2 and 3 studied later. 
In this rectangular domain case, $\Omega$ has the Fourier expansion

$$
\Omega=\sum_{k \in \mathbb{Z}^{2} /\{0\}} \omega_{k} e^{i\left[\alpha k_{1} x_{1}+k_{2} x_{2}\right]}
$$

We have equation (4.3) with

$$
a_{n}=-\frac{4}{\alpha \hat{k}_{1}} \frac{\left(\alpha \hat{k}_{1}\right)^{2}+\left(\hat{k}_{2}+n\right)^{2}}{\left(\alpha \hat{k}_{1}\right)^{2}+\left(\hat{k}_{2}+n\right)^{2}-1}\left\{\lambda+\nu\left[\left(\alpha \hat{k}_{1}\right)^{2}+\left(\hat{k}_{2}+n\right)^{2}\right]\right\} .
$$

THEOREM 4.1. The spectra of the 2D linear Euler operator $L$ have the following properties.

(1) $\left(\alpha \hat{k}_{1}\right)^{2}+\left(\hat{k}_{2}+n\right)^{2}>1, \forall n \in \mathbb{Z}$. When $\nu \rightarrow 0$, there is no eigenvalue of non-negative real part. When $\nu=0$, the entire spectrum is the continuous spectrum

$$
\left[-i \frac{\alpha\left|\hat{k}_{1}\right|}{2}, i \frac{\alpha\left|\hat{k}_{1}\right|}{2}\right] .
$$

(2) $\hat{k}_{1}=0, \hat{k}_{2}=1$. The spectrum consists of the eigenvalues

$$
\lambda=-\nu n^{2}, \quad n \in \mathbb{Z} /\{0\} .
$$

The eigenfunctions are the Fourier modes

$$
\tilde{\omega}_{n p} e^{i n x_{2}}+\text { c.c. }, \quad \forall \tilde{\omega}_{n p} \in \mathbb{C}, \quad n \in \mathbb{Z} /\{0\} .
$$

As $\nu \rightarrow 0^{+}$, the eigenvalues are dense on the negative half of the real axis $(-\infty, 0]$. Setting $\nu=0$, the only eigenvalue is $\lambda=0$ of infinite multiplicity with the same eigenfunctions as above.

(3) $\hat{k}_{1}=-1, \hat{k}_{2}=0$. (a). $\nu>0$. For any $\alpha \in(0.5,0.95)$, there is a unique $\nu_{*}(\alpha)$,

$$
\frac{\sqrt{32-3 \alpha^{6}-17 \alpha^{4}-16 \alpha^{2}}}{4\left(\alpha^{2}+1\right)\left(\alpha^{2}+4\right)}<\nu_{*}(\alpha)<\frac{1}{2\left(\alpha^{2}+1\right)} \sqrt{\frac{1-\alpha^{2}}{2}}
$$

where the term under the square root on the left is positive for $\alpha \in$ $(0.5,0.95)$, and the left term is always less than the right term. When $\nu>\nu_{*}(\alpha)$, there is no eigenvalue of non-negative real part. When $\nu=$ $\nu_{*}(\alpha), \lambda=0$ is an eigenvalue, and all the rest eigenvalues have negative real parts. When $\nu<\nu_{*}(\alpha)$, there is a unique positive eigenvalue $\lambda(\nu)>0$, and all the rest eigenvalues have negative real parts. $\nu^{-1} \lambda(\nu)$ is a strictly monotonically decreasing function of $\nu$. When $\alpha \in(0.5,0.8469)$, we have the estimate

$$
\begin{aligned}
& \sqrt{\frac{\alpha^{2}\left(1-\alpha^{2}\right)}{8\left(\alpha^{2}+1\right)}-\frac{\alpha^{4}\left(\alpha^{2}+3\right)}{16\left(\alpha^{2}+1\right)\left(\alpha^{2}+4\right)}}-\nu\left(\alpha^{2}+1\right)<\lambda(\nu) \\
& <\sqrt{\frac{\alpha^{2}\left(1-\alpha^{2}\right)}{8\left(\alpha^{2}+1\right)}}-\nu \alpha^{2},
\end{aligned}
$$

where the term under the square root on the left is positive for $\alpha \in$ $(0.5,0.8469)$.

$$
\sqrt{\frac{\alpha^{2}\left(1-\alpha^{2}\right)}{8\left(\alpha^{2}+1\right)}-\frac{\alpha^{4}\left(\alpha^{2}+3\right)}{16\left(\alpha^{2}+1\right)\left(\alpha^{2}+4\right)}} \leq \lim _{\nu \rightarrow 0^{+}} \lambda(\nu) \leq \sqrt{\frac{\alpha^{2}\left(1-\alpha^{2}\right)}{8\left(\alpha^{2}+1\right)}} .
$$


In particular, as $\nu \rightarrow 0^{+}, \lambda(\nu)=O(1)$.

(b). $\nu=0$. When $\alpha \in(0.5,0.8469)$, we have only two eigenvalues $\lambda_{0}$ and $-\lambda_{0}$, where $\lambda_{0}$ is positive,

$$
\sqrt{\frac{\alpha^{2}\left(1-\alpha^{2}\right)}{8\left(\alpha^{2}+1\right)}-\frac{\alpha^{4}\left(\alpha^{2}+3\right)}{16\left(\alpha^{2}+1\right)\left(\alpha^{2}+4\right)}}<\lambda_{0}<\sqrt{\frac{\alpha^{2}\left(1-\alpha^{2}\right)}{8\left(\alpha^{2}+1\right)}} .
$$

The rest of the spectrum is a continuous spectrum $[-i \alpha / 2, i \alpha / 2]$.

(c). For any fixed $\alpha \in(0.5,0.8469)$,

$$
\lim _{\nu \rightarrow 0^{+}} \lambda(\nu)=\lambda_{0} \text {. }
$$

(4) Finally, when $\nu=0$, the union of all the above pieces of continuous spectra is the imaginary axis $i \mathbb{R}$.

From Theorems 3.5 and 4.1, we have the corollary.

Corollary 4.2. For any $\alpha \in(0.5,0.95)$, and $\nu \in\left(0, \nu_{*}(\alpha)\right)$ where $\nu_{*}(\alpha)>0$ satisfies (4.9), in a neighborhood of $\Omega_{*}$ in the Sobolev space $H^{\ell}\left(\mathbb{T}^{2}\right)(\ell \geq 3)$, there are an 1-dimensional $C^{\infty}$ unstable manifold and an 1-codimensional $C^{\infty}$ stable manifold.

Remark 4.3. In the Theorem 4.1, (4.10) verifies part of Assumption 1. That is, $\operatorname{Re}\left\{\lambda_{1}^{u}\right\}$ is of order $O(1)$ as $\nu \rightarrow 0^{+}$. Case 2 of Theorem 4.1 indicates that $\operatorname{Re}\left\{\lambda_{1}^{s}\right\}$ is at least $O(\nu)$ as $\nu \rightarrow 0^{+}$. Case 3 indicates that the constant $C$ in (3.7) and (3.11) should be $O(1)$ as $\nu \rightarrow 0^{+}$.

Proof. We will give the proof case by case.

(1) The case (a). $\nu>0$. If $\operatorname{Re}\{\lambda\} \geq 0$, then all the $\operatorname{Re}\left\{a_{n}\right\}$ 's are nonzero and have the same sign. The real parts of the right and left hand sides of (4.8) are nonzero but of different signs. Thus there is no eigenvalue of non-negative real part. The case (b). $\nu=0$. If $\operatorname{Re}\{\lambda\} \neq 0$, then all the $\operatorname{Re}\left\{a_{n}\right\}$ 's are nonzero and have the same sign. Again (4.8) can not be satisfied. If $\operatorname{Re}\{\lambda\}=0$, let $\tilde{a}=\lim _{n \rightarrow \infty} a_{n}=-\frac{4}{\alpha \hat{k}_{1}} \lambda$, then

$$
\tilde{a} z_{n}=\frac{\left(\alpha \hat{k}_{1}\right)^{2}+\left(\hat{k}_{2}+n\right)^{2}-1}{\left(\alpha \hat{k}_{1}\right)^{2}+\left(\hat{k}_{2}+n\right)^{2}}\left(z_{n+1}-z_{n-1}\right),
$$

where $\left(\alpha \hat{k}_{1}\right)^{2}+\left(\hat{k}_{2}+n\right)^{2}>1, \forall n \in \mathbb{Z}$. By using $\ell_{2}$ norm of $\left\{z_{n}\right\}_{n \in \mathbb{Z}}$, one sees that possible eigenvalues have to satisfy $|\tilde{a}| \leq 2$. As mentioned before, $\operatorname{Re}\{\lambda\}=0$ and $|\tilde{a}| \leq 2$ correspond to a continuous spectrum [9], which is the interval

$$
\left[-i \frac{\alpha\left|\hat{k}_{1}\right|}{2}, i \frac{\alpha\left|\hat{k}_{1}\right|}{2}\right]
$$

(2) In this case, one has

$$
\left[\lambda+\nu(n+1)^{2}\right] \tilde{\omega}_{(n+1) p}=0 .
$$

The claims follow immediately.

(3) In this case,

$$
a_{n}=\frac{4}{\alpha} \frac{\alpha^{2}+n^{2}}{\alpha^{2}+n^{2}-1}\left[\lambda+\nu\left(\alpha^{2}+n^{2}\right)\right] .
$$


Thus $a_{-n}=a_{n}$. Equation (4.8) is reduced to

$$
-a_{0} / 2=\frac{1}{a_{1}+\frac{1}{a_{2}+\cdots}} .
$$

The first a few $a_{n}$ 's are

$$
\begin{aligned}
& a_{0}=\frac{4}{\alpha} \frac{\alpha^{2}}{\alpha^{2}-1}\left[\lambda+\nu \alpha^{2}\right], \\
& a_{1}=\frac{4}{\alpha} \frac{\alpha^{2}+1}{\alpha^{2}}\left[\lambda+\nu\left(\alpha^{2}+1\right)\right], \\
& a_{2}=\frac{4}{\alpha} \frac{\alpha^{2}+4}{\alpha^{2}+3}\left[\lambda+\nu\left(\alpha^{2}+4\right)\right] .
\end{aligned}
$$

Let

$$
f(\lambda)=\frac{1}{a_{1}+\frac{1}{a_{2}+\cdots}}, \quad g(\lambda)=-a_{0} / 2 .
$$

(a). $\nu>0$. First we show that if $\lambda(\operatorname{Re}\{\lambda\} \geq 0)$ is a solution of (4.12), then $\lambda$ must be real. Assume $\operatorname{Im}\{\lambda\}>0$, then

$$
\arg \left(-a_{0}\right)>\arg \left(a_{1}\right)>\arg \left(a_{2}\right)>\cdots \geq 0 .
$$

Thus

$$
|\arg (f(\lambda))| \leq \arg \left(a_{1}\right)
$$

But

$$
\arg (g(\lambda))=\arg \left(-a_{0}\right)>|\arg (f(\lambda))|,
$$

a contradiction. Similarly for the case $\operatorname{Im}\{\lambda\}<0$, thus $\lambda(\operatorname{Re}\{\lambda\} \geq 0)$ is real. Next we show that there is a $\nu_{0}>0$ such that for every $\nu \leq \nu_{0}$, there is a unique eigenvalue $\lambda>0$. Since $a_{n}>0, \forall n \geq 1$, we have

$$
\frac{1}{a_{1}+\frac{1}{a_{2}}}<f(\lambda)<\frac{1}{a_{1}} \text {. }
$$

Thus when $\lambda$ is large enough

$$
f(\lambda)<g(\lambda)
$$

From (4.13),

$$
f(0)>\frac{1}{\frac{4 \nu\left(\alpha^{2}+1\right)^{2}}{\alpha^{3}}+\frac{\alpha\left(\alpha^{2}+3\right)}{4 \nu\left(\alpha^{2}+4\right)^{2}}} .
$$

We know that

$$
g(0)=\frac{2 \nu \alpha^{3}}{1-\alpha^{2}} .
$$

We want to choose $\nu$ such that

$$
\frac{1}{\frac{4 \nu\left(\alpha^{2}+1\right)^{2}}{\alpha^{3}}+\frac{\alpha\left(\alpha^{2}+3\right)}{4 \nu\left(\alpha^{2}+4\right)^{2}}} \geq \frac{2 \nu \alpha^{3}}{1-\alpha^{2}} .
$$

Thus

$$
\nu \leq \nu_{0}(\alpha)=\frac{\sqrt{32-3 \alpha^{6}-17 \alpha^{4}-16 \alpha^{2}}}{4\left(\alpha^{2}+1\right)\left(\alpha^{2}+4\right)} \quad\left(1 / 2<\alpha<\alpha_{0}\right)
$$

where $0.95<\alpha_{0}<1$ and $\alpha_{0}$ satisfies

$$
32-3 \alpha^{6}-17 \alpha^{4}-16 \alpha^{2}=0 .
$$


For example,

$$
\nu_{0}(0.5) \approx 0.244, \quad \nu_{0}(0.95) \approx 0.0329 .
$$

For each fixed $\nu, \nu \leq \nu_{0}(\alpha)$, we have

$$
f(0)>g(0) \text {. }
$$

From (4.14) and (4.16), we see that there is a $\lambda>0$ such that (4.12) is true. Next, we want to show that this eigenvalue is unique. Notice that

$$
\begin{aligned}
& \left(\lambda+\nu \alpha^{2}\right)^{-1} g(\lambda)=\frac{2 \alpha}{1-\alpha^{2}}, \\
& \left(\lambda+\nu \alpha^{2}\right)^{-1} f(\lambda)=\frac{1}{\left(\lambda+\nu \alpha^{2}\right) a_{1}+\frac{1}{\left(\lambda+\nu \alpha^{2}\right)^{-1} a_{2}+\cdots}} .
\end{aligned}
$$

Since $\left(\lambda+\nu \alpha^{2}\right) a_{2 n+1}(n \geq 0)$ is a strictly monotonically increasing function of $\lambda$ for $\lambda>0$, and $\left(\lambda+\nu \alpha^{2}\right)^{-1} a_{2 n}(n \geq 1)$ is a strictly monotonically decreasing function of $\lambda$ for $\lambda>0$, we see that $\left(\lambda+\nu \alpha^{2}\right)^{-1} f(\lambda)$ is a strictly monotonically decreasing function of $\lambda$ for $\lambda>0$. Thus the eigenvalue which satisfies

$$
\left(\lambda+\nu \alpha^{2}\right)^{-1} f(\lambda)=\left(\lambda+\nu \alpha^{2}\right)^{-1} g(\lambda)
$$

is unique. Similarly, $\nu \alpha^{2} a_{2 n+1}(n \geq 0)$ is a strictly monotonically increasing function of $\nu$ for $\nu>0$, and $\left(\nu \alpha^{2}\right)^{-1} a_{2 n}(n \geq 1)$ is a constant function of $\nu$. Then $\left(\nu \alpha^{2}\right)^{-1} f(0)$ is a strictly monotonically decreasing function of $\nu$ for $\nu>0$. We know from above that when $\nu=\nu_{0}(\alpha)$,

$$
\left(\nu \alpha^{2}\right)^{-1} f(0)>\left(\nu \alpha^{2}\right)^{-1} g(0)=\frac{2 \alpha}{1-\alpha^{2}}, \quad \text { constant in } \nu .
$$

From (4.13), we have

$$
\left(\nu \alpha^{2}\right)^{-1} f(0)<\frac{1}{\left(\nu \alpha^{2}\right) a_{1}}=\frac{\alpha}{4\left(\alpha^{2}+1\right)^{2}} \frac{1}{\nu^{2}},
$$

thus when $\nu>0$ is large enough

$$
\left(\nu \alpha^{2}\right)^{-1} f(0)<\left(\nu \alpha^{2}\right)^{-1} g(0) .
$$

Therefore, there is a unique $\nu_{*}(\alpha)>\nu_{0}(\alpha)$, such that

$$
\left(\nu_{*} \alpha^{2}\right)^{-1} f(0)=\left(\nu_{*} \alpha^{2}\right)^{-1} g(0) .
$$

Thus we have shown the following claims: When $\nu>\nu_{*}(\alpha)$, there is no eigenvalue of non-negative real part. When $\nu=\nu_{*}(\alpha), \lambda=0$ is an eigenvalue, and all the rest eigenvalues have negative real parts. When $\nu<\nu_{*}(\alpha)$, there is a unique positive eigenvalue $\lambda(\nu)>0$, and all the rest eigenvalues have negative real parts. An estimate for $\nu_{*}$ can be obtained from (4.13),

$$
\frac{1}{\frac{4\left(\alpha^{2}+1\right)^{2}}{\alpha} \nu_{*}^{2}+\frac{\alpha^{3}\left(\alpha^{2}+3\right)}{4\left(\alpha^{2}+4\right)^{2}}}<\frac{2 \alpha}{1-\alpha^{2}}<\frac{1}{\frac{4\left(\alpha^{2}+1\right)^{2}}{\alpha} \nu_{*}^{2}} .
$$

We have

$$
\nu_{0}(\alpha)<\nu_{*}(\alpha)<\frac{1}{2\left(\alpha^{2}+1\right)} \sqrt{\frac{1-\alpha^{2}}{2}},
$$


where $\nu_{0}(\alpha)$ is given by (4.15). For example,

$$
0.244029<\nu_{*}(0.5)<0.244949, \quad 0.0329<\nu_{*}(0.95)<0.058 .
$$

Next we want to show that the unique eigenvalue $\lambda(\nu)$ has the property that $\nu^{-1} \lambda(\nu)$ is a strictly monotonically decreasing function of $\nu$. Notice that

$$
\begin{aligned}
\left(\lambda+\nu \alpha^{2}\right) a_{2 n+1}= & \frac{4}{\alpha} \frac{\alpha^{2}+(2 n+1)^{2}}{\alpha^{2}+(2 n+1)^{2}-1} \nu^{2}\left(\frac{\lambda}{\nu}+\alpha^{2}\right) \\
& \left(\frac{\lambda}{\nu}+\left[\alpha^{2}+(2 n+1)^{2}\right]\right), \quad(n \geq 0), \\
\left(\lambda+\nu \alpha^{2}\right)^{-1} a_{2 n}= & \frac{4}{\alpha} \frac{\alpha^{2}+(2 n)^{2}}{\alpha^{2}+(2 n)^{2}-1}\left[1+\frac{(2 n)^{2}}{\frac{\lambda}{\nu}+\alpha^{2}}\right], \quad(n \geq 1) .
\end{aligned}
$$

Assume that $\nu^{-1} \lambda(\nu)$ is not a strictly monotonically decreasing function of $\nu$, then there is an interval in which $\nu^{-1} \lambda(\nu)$ is a strictly monotonically increasing or a constant function of $\nu$. In that interval, $\left[\lambda(\nu)+\nu \alpha^{2}\right] a_{2 n+1}$ $(n \geq 0)$ is strictly monotonically increasing, and $\left[\lambda(\nu)+\nu \alpha^{2}\right]^{-1} a_{2 n}(n \geq 1)$ is monotonically decreasing. Thus $\left[\lambda(\nu)+\nu \alpha^{2}\right]^{-1} f(\lambda(\nu))$ is a strictly monotonically decreasing function of $\nu$. On the other hand,

$$
\left[\lambda(\nu)+\nu \alpha^{2}\right]^{-1} g(\lambda(\nu))=\frac{2 \alpha}{1-\alpha^{2}}
$$

is a constant function of $\nu$. This contradiction shows that $\nu^{-1} \lambda(\nu)$ is a strictly monotonically decreasing function of $\nu$. Next we want to show that $\lambda(\nu)=O(1)$ as $\nu \rightarrow 0^{+}$. From (4.12) and (4.13), we have

$$
\begin{gathered}
\frac{1}{\frac{4\left(\alpha^{2}+1\right)\left[\lambda+\nu\left(\alpha^{2}+1\right)\right]}{\alpha^{3}}+\frac{\alpha\left(\alpha^{2}+3\right)}{4\left(\alpha^{2}+4\right)\left[\lambda+\nu\left(\alpha^{2}+4\right)\right]}} \\
<\frac{2 \alpha}{1-\alpha^{2}}\left(\lambda+\nu \alpha^{2}\right)<\frac{\alpha^{3}}{4\left(\alpha^{2}+1\right)\left[\lambda+\nu\left(\alpha^{2}+1\right)\right]} .
\end{gathered}
$$

Let $\nu \rightarrow 0^{+}$, we have

$$
\sqrt{\frac{\alpha^{2}\left(1-\alpha^{2}\right)}{8\left(\alpha^{2}+1\right)}-\frac{\alpha^{4}\left(\alpha^{2}+3\right)}{16\left(\alpha^{2}+1\right)\left(\alpha^{2}+4\right)}} \leq \lambda \leq \sqrt{\frac{\alpha^{2}\left(1-\alpha^{2}\right)}{8\left(\alpha^{2}+1\right)}} .
$$

For the term under the square root on the left to be positive, we need

$$
\alpha<\alpha_{1}=\sqrt{\sqrt{\frac{59}{12}}-\frac{3}{2}} \approx 0.8469 .
$$

Thus when $\alpha \in\left(1 / 2, \alpha_{1}\right)$,

$$
\lambda(\nu)=O(1), \quad \text { as } \nu \rightarrow 0^{+} .
$$

Also from (4.20), we have the estimate

$$
\begin{aligned}
& \sqrt{\frac{\alpha^{2}\left(1-\alpha^{2}\right)}{8\left(\alpha^{2}+1\right)}-\frac{\alpha^{4}\left(\alpha^{2}+3\right)}{16\left(\alpha^{2}+1\right)\left(\alpha^{2}+4\right)}}-\nu\left(\alpha^{2}+1\right)<\lambda(\nu) \\
< & \sqrt{\frac{\alpha^{2}\left(1-\alpha^{2}\right)}{8\left(\alpha^{2}+1\right)}}-\nu \alpha^{2} .
\end{aligned}
$$


(b). $\nu=0$. We have

$$
a_{n}=\frac{4}{\alpha} \frac{\alpha^{2}+n^{2}}{\alpha^{2}+n^{2}-1} \lambda .
$$

As $|n| \rightarrow \infty, a_{n} \rightarrow \tilde{a}=\frac{4}{\alpha} \lambda$. As before, $\operatorname{Re}\{\lambda\}=0$ and $|\lambda| \leq \frac{\alpha}{2}$ corresponds to a continuous spectrum. Next we show that outside the disc $|\lambda| \leq \frac{\alpha}{2}$, there is no eigenvalue. Outside the disc, $|\tilde{a}|>2$,

$$
\begin{gathered}
\left|a_{0}\right|>2 \frac{\alpha^{2}}{1-\alpha^{2}}>\frac{2}{3}, \quad \text { for } \alpha \in(1 / 2,1), \\
\left|a_{1}\right|>2\left(1+\frac{1}{\alpha^{2}}\right)>4, \quad \text { for } \alpha \in(1 / 2,1),
\end{gathered}
$$

and

$$
\left|a_{n}\right|>2, \quad \forall n \geq 2 .
$$

By a result of continued fraction [15],

$$
\left|\frac{1}{a_{2}+\frac{1}{a_{3}+\cdots}}\right| \leq 1 \text {. }
$$

Thus

$$
|g(\lambda)|>\frac{1}{3}
$$

while

$$
|f(\lambda)| \leq \frac{1}{\left|a_{1}\right|-\left|\frac{1}{a_{2}+\frac{1}{a_{3}+\cdots}}\right|}<\frac{1}{3} .
$$

This contradiction proves the claim. Thus the possible eigenvalues should lie in the region $\operatorname{Re}\{\lambda\} \neq 0$ and $|\lambda| \leq \frac{\alpha}{2}$. Since the eigenvalues are symmetric with respect to the imaginary axis [9], we only need to consider the case $\operatorname{Re}\{\lambda\}>0$. First we show that the possible eigenvalue must be real. Assume that $\operatorname{Im}\{\lambda\}>0$, then

$$
\arg \left(-a_{0}\right)=\arg \left(a_{1}\right)=\arg \left(a_{2}\right)=\cdots>0 .
$$

Thus

Then

$$
\left|\arg \left(\frac{1}{a_{2}+\frac{1}{a_{3}+\cdots}}\right)\right| \leq \arg \left(a_{1}\right) .
$$

Therefore

$$
\arg \left(a_{1}+\frac{1}{a_{2}+\frac{1}{a_{3}+\cdots}}\right) \neq-\arg \left(a_{1}\right) .
$$

$$
\arg (f(\lambda)) \neq \arg \left(a_{1}\right)=\arg \left(-a_{0}\right)=\arg (g(\lambda)) .
$$

This is a contradiction. The case $\operatorname{Im}\{\lambda\}<0$ is similar, and the claim is proved. When $\lambda>0$,

$$
\frac{1}{\lambda a_{1}+\frac{1}{\lambda^{-1} a_{2}}}<\lambda^{-1} f(\lambda)<\frac{1}{\lambda a_{1}},
$$

i.e.

$$
\frac{1}{\frac{4\left(\alpha^{2}+1\right)}{\alpha^{3}} \lambda^{2}+\frac{\alpha\left(\alpha^{2}+3\right)}{4\left(\alpha^{2}+4\right)}}<\lambda^{-1} f(\lambda)<\frac{1}{\frac{4\left(\alpha^{2}+1\right)}{\alpha^{3}} \lambda^{2}} .
$$


Thus

$$
\lim _{\lambda \rightarrow+\infty} \lambda^{-1} f(\lambda)=0, \quad \lim _{\lambda \rightarrow 0^{+}} \lambda^{-1} f(\lambda) \geq \frac{4\left(\alpha^{2}+4\right)}{\alpha\left(\alpha^{2}+3\right)} .
$$

Notice that

$$
\lambda^{-1} g(\lambda)=\frac{2 \alpha}{1-\alpha^{2}} .
$$

We want to choose $\alpha$ such that

$$
\frac{2 \alpha}{1-\alpha^{2}}<\frac{4\left(\alpha^{2}+4\right)}{\alpha\left(\alpha^{2}+3\right)} .
$$

This condition is equivalent to the fact that the term under the square root on the left of (4.21) being positive. Thus we have

$$
\alpha<\alpha_{1}=\sqrt{\sqrt{\frac{59}{12}}-\frac{3}{2}} \approx 0.8469
$$

which is the same with (4.22). Thus when $\alpha \in\left(1 / 2, \alpha_{1}\right)$, there is a positive eigenvalue $\lambda_{0}$,

$$
\lambda_{0}^{-1} f\left(\lambda_{0}\right)=\lambda_{0}^{-1} g\left(\lambda_{0}\right)=\frac{2 \alpha}{1-\alpha^{2}} .
$$

Since $\lambda a_{2 n+1}(n \geq 0)$ is a strictly monotonically increasing function of $\lambda$ for $\lambda>0$, and $\lambda^{-1} a_{2 n}(n \geq 1)$ is a constant function of $\lambda$, we see that $\lambda^{-1} f(\lambda)$ is a strictly monotonically decreasing function of $\lambda$ for $\lambda>0$. Thus the positive eigenvalue $\lambda_{0}$ is unique. From (4.24), we have

$$
\frac{1}{\frac{4\left(\alpha^{2}+1\right)}{\alpha^{3}} \lambda_{0}^{2}+\frac{\alpha\left(\alpha^{2}+3\right)}{4\left(\alpha^{2}+4\right)}}<\frac{2 \alpha}{1-\alpha^{2}}<\frac{1}{\frac{4\left(\alpha^{2}+1\right)}{\alpha^{3}} \lambda_{0}^{2}},
$$

i.e.

$$
\sqrt{\frac{\alpha^{2}\left(1-\alpha^{2}\right)}{8\left(\alpha^{2}+1\right)}-\frac{\alpha^{4}\left(\alpha^{2}+3\right)}{16\left(\alpha^{2}+1\right)\left(\alpha^{2}+4\right)}}<\lambda_{0}<\sqrt{\frac{\alpha^{2}\left(1-\alpha^{2}\right)}{8\left(\alpha^{2}+1\right)}}
$$

which is similar to (4.21). Next we want to show that

$$
\lim _{\nu \rightarrow 0^{+}} \lambda(\nu)=\lambda_{0} \text {. }
$$

Let $F(\nu, \lambda)=f(\lambda)-g(\lambda)$, and let $F_{N}(\nu, \lambda)$ be the $N$-th truncation of $F(\nu, \lambda)$,

Notice that

$$
F_{N}(\nu, \lambda)=\frac{a_{0}}{2}+\frac{1}{a_{1}+\frac{1}{a_{2}+\cdots+\frac{1}{a_{n}}}}
$$

$$
\begin{gathered}
F\left(\nu, \lambda(\nu)=0, \quad \nu \in\left(0, \nu_{*}(\alpha)\right), \quad \alpha \in(0.5,0.8469) ;\right. \\
F\left(0, \lambda_{0}\right)=0, \quad \alpha \in(0.5,0.8469) ;
\end{gathered}
$$

where $\lambda(\nu)$ and $\lambda_{0}$ have the estimates (4.23) and (4.25). Thus for any fixed $\alpha \in(0.5,0.8469),(\nu, \lambda(\nu))$ lies in a compact set $[0, \delta] \times\left[c_{1}, c_{2}\right]$ where $\delta, c_{1}$ and $c_{2}$ are positive constants. Assume that (4.26) is not true, then there is a sequence $\left(\nu_{j}, \lambda\left(\nu_{j}\right)\right)$ such that

$$
\lim _{\nu_{j} \rightarrow 0^{+}} \lambda\left(\nu_{j}\right)=\tilde{\lambda}_{0}
$$


where

$$
\tilde{\lambda}_{0} \neq \lambda_{0} \quad \text { and } \quad \tilde{\lambda}_{0} \in\left[c_{1}, c_{2}\right] .
$$

By a result of continued fraction $[6]$,

$$
\lim _{N \rightarrow \infty} F_{N}(\nu, \lambda)=F(\nu, \lambda)
$$

uniformly for $\nu \in[0, \delta]$ and $\lambda \in\left[c_{1}, c_{2}\right]$. Thus for any $\epsilon>0$, there is an integer $N_{0}$ such that for any $N \geq N_{0}$,

$$
\left|F_{N}\left(\nu_{j}, \lambda\left(\nu_{j}\right)\right)-F\left(\nu_{j}, \lambda\left(\nu_{j}\right)\right)\right|<\epsilon,
$$

i.e.

$$
\left|F_{N}\left(\nu_{j}, \lambda\left(\nu_{j}\right)\right)\right|<\epsilon, \quad \forall j .
$$

For any fixed $N$, let $j \rightarrow+\infty$, we have

$$
\left|F_{N}\left(0, \tilde{\lambda}_{0}\right)\right| \leq \epsilon .
$$

Let $N \rightarrow+\infty$, we get

$$
\left|F\left(0, \tilde{\lambda}_{0}\right)\right| \leq \epsilon .
$$

Since $\epsilon$ is arbitrarily small, we have

$$
F\left(0, \tilde{\lambda}_{0}\right)=0 .
$$

By the uniqueness of the eigenvalue, $\tilde{\lambda}_{0}=\lambda_{0}$. This contradiction shows that (4.26) is true.

(4) When $\nu=0$, the union of all the continuous spatra

$$
\left[-i \frac{\alpha\left|\hat{k}_{1}\right|}{2}, i \frac{\alpha\left|\hat{k}_{1}\right|}{2}\right],
$$

is $i \mathbb{R}$.

4.2. Example 2. Our second prime example is $\Omega_{*}=\cos \left(x_{1}+x_{2}\right)$ which corresponds to $p=(1,1)^{T}$ and $\Gamma=1 / 2$ in $(4.1)$. Here

$$
\begin{aligned}
a_{n}= & \frac{8}{\left(\hat{k}_{2}-\hat{k}_{1}\right)} \frac{\left(\hat{k}_{1}+n\right)^{2}+\left(\hat{k}_{2}+n\right)^{2}}{\left(\hat{k}_{1}+n\right)^{2}+\left(\hat{k}_{2}+n\right)^{2}-2} \\
& \times\left\{\lambda+\nu\left[\left(\hat{k}_{1}+n\right)^{2}+\left(\hat{k}_{2}+n\right)^{2}\right]\right\} .
\end{aligned}
$$

This example has two possible unstable eigenvalues. For shears, the number of unstable eigenvalues of 2D linear Euler is bounded by the number of lattices points inside the disc of radius $|p|[\mathbf{8}]$, which is even for square lattice. In [13] [14], Vincent Liu studied another shear which has three possible unstable eigenvalues. Liu did a detailed calculation on the eigenvalues, which will be discussed in next subsection. Here we will do an even more detailed calculation.

THEOREM 4.4. The spectra of the 2D linear Euler operator L have the following properties. 
(1) The set $\{\hat{k}+n p\}_{n \in \mathbb{Z}}$ has no intersection with the disc of radius $|p|=\sqrt{2}$. When $\nu>0$, there is no eigenvalue of non-negative real part. When $\nu=0$, the entire spectrum is the continuous spectrum

$$
\left[-i \frac{1}{4}\left|\hat{k}_{2}-\hat{k}_{1}\right|, \quad i \frac{1}{4}\left|\hat{k}_{2}-\hat{k}_{1}\right|\right] \text {. }
$$

(2) $\hat{k}=p=(1,1)^{T}$. The spectrum consists of the eigenvalues

$$
\lambda=-\nu 2 n^{2}, \quad n \in \mathbb{Z} /\{0\} .
$$

The eigenfunctions are the Fourier modes

$$
\tilde{\omega}_{n p} e^{i n p \cdot x}+\text { c.c. }, \quad \forall \tilde{\omega}_{n p} \in \mathbb{C}, \quad n \in \mathbb{Z} /\{0\} .
$$

As $\nu \rightarrow 0^{+}$, the eigenvalues are dense on the negative half of the real axis $(-\infty, 0]$. Setting $\nu=0$, the only eigenvalue is $\lambda=0$ of infinite multiplicity with the same eigenfunctions as above.

(3) $\hat{k}=(-1,1)^{T}$. When $\nu>0$, there is no eigenvalue of non-negative real part. When $\nu=0$, the entire spectrum is the continuous spectrum $\left[-i \frac{1}{2}, i \frac{1}{2}\right]$. A special eigenvalue is $\lambda=-2 \nu$ (when $\nu=0$, this eigenvalue $\lambda=0$ is embedded in the continuous spectrum).

(4) $\hat{k}=(0,1)^{T}$. When $\nu>0$, in the half plane $\operatorname{Re}\{\lambda\} \geq-\nu$, there is a unique pair of eigenvalues $\lambda$ and $\bar{\lambda}$ such that

$$
\begin{gathered}
-\nu<\operatorname{Re}\{\lambda\}<\frac{1}{4} \sqrt{\frac{3}{20}+(8 \nu)^{2}}-2 \nu, \\
\frac{1}{8}\left(1-\sqrt{\frac{3}{5}}\right)<\operatorname{Im}\{\lambda\}<\frac{1}{8}\left(1+\sqrt{\frac{3}{5}}\right) .
\end{gathered}
$$

When $\nu=0,\left[-i \frac{1}{4}, i \frac{1}{4}\right]$ is a continuous spectrum. If there is an eigenvalue of positive real part, then there is a quadruplet $(\lambda, \bar{\lambda}-\lambda-\bar{\lambda})$ where

$$
\begin{gathered}
0<\operatorname{Re}\{\lambda\}<\frac{1}{16} \sqrt{\frac{3}{5}}, \\
\frac{1}{8}\left(1-\sqrt{\frac{3}{5}}\right)<\operatorname{Im}\{\lambda\}<\frac{1}{8}\left(1+\sqrt{\frac{3}{5}}\right) .
\end{gathered}
$$

(5) Finally, when $\nu=0$, the union of all the above pieces of continuous spectra is the imaginary axis $i \mathbb{R}$.

Remark 4.5. For Case 4, numerical computation indicates that when $\nu>0$, the real part of the eigenvalue $\operatorname{Re}\{\lambda(\nu)\} \geq c>0$, where $c$ is independent of $\nu$. When $\nu=0$, numerical computation indicates that the eigenvalue $\lambda(0)$ indeed exists and $\operatorname{Re}\{\lambda(0)\} \geq c>0$ (the same $c$ as above). Numerical computation also indicates that as $\nu \rightarrow 0^{+}, \lambda(\nu) \rightarrow \lambda(0)$ which can be proved given the above facts. Cases 2 and 3 indicate that $\operatorname{Re}\left\{\lambda_{1}^{s}\right\}$ in Assumption 1 is at least $O(\nu)$ as $\nu \rightarrow 0^{+}$.

Proof. We will give the proof case by case.

(1) The case (a). $\nu>0$. If $\operatorname{Re}\{\lambda\} \geq 0$, then all the $\operatorname{Re}\left\{a_{n}\right\}$ 's are nonzero and have the same sign. The real parts of the right and left hand sides of (4.8) are nonzero but of different signs. Thus there is no eigenvalue of non-negative real part. The case (b). $\nu=0$. If $\operatorname{Re}\{\lambda\} \neq 0$, then all 
the $\operatorname{Re}\left\{a_{n}\right\}$ 's are nonzero and have the same sign. Again (4.8) can not be satisfied. If $\operatorname{Re}\{\lambda\}=0$, let $\tilde{a}=\lim _{n \rightarrow \infty} a_{n}=\frac{8}{\hat{k}_{2}-\hat{k}_{1}} \lambda$, then

$$
\tilde{a} z_{n}=\frac{|\hat{k}+n p|^{2}-2}{|\hat{k}+n p|^{2}}\left(z_{n+1}-z_{n-1}\right),
$$

where $0<|\hat{k}+n p|^{2}-2<|\hat{k}+n p|^{2}, \forall n \in \mathbb{Z}$. By using $\ell_{2}$ norm of $\left\{z_{n}\right\}_{n \in \mathbb{Z}}$, one sees that possible eigenvalues have to satisfy $|\tilde{a}| \leq 2$. As mentioned before, $\operatorname{Re}\{\lambda\}=0$ and $|\tilde{a}| \leq 2$ correspond to a continuous spectrum [9], which is the interval

$$
\left[-i \frac{1}{4}\left|\hat{k}_{2}-\hat{k}_{1}\right|, i \frac{1}{4}\left|\hat{k}_{2}-\hat{k}_{1}\right|\right] \text {. }
$$

(2) In this case, one has

$$
\left[\lambda+\nu 2(n+1)^{2}\right] \tilde{\omega}_{(n+1) p}=0 .
$$

The claims follow immediately.

(3) In this case, denote $\tilde{\omega}_{\hat{k}+n p}$ simply by $\omega_{n}$, one has

$$
\left[\lambda+2 \nu\left(n^{2}+1\right)\right] \omega_{n}=\frac{1}{4} \frac{(n+1)^{2}}{(n+1)^{2}+1} \omega_{n+1}-\frac{1}{4} \frac{(n-1)^{2}}{(n-1)^{2}+1} \omega_{n-1} .
$$

This system decouples at $n=0$. A few equations around $n=0$ are

$$
\begin{aligned}
(\lambda+10 \nu) \omega_{-2} & =\frac{1}{8} \omega_{-1}-\frac{9}{40} \omega_{-3}, \\
(\lambda+4 \nu) \omega_{-1} & =-\frac{1}{5} \omega_{-2} \\
(\lambda+2 \nu) \omega_{0} & =\frac{1}{8} \omega_{1}-\frac{1}{8} \omega_{-1}, \\
(\lambda+4 \nu) \omega_{1} & =\frac{1}{5} \omega_{2} \\
(\lambda+10 \nu) \omega_{2} & =\frac{9}{40} \omega_{3}-\frac{1}{8} \omega_{1} .
\end{aligned}
$$

Notice that

$$
a_{n}=4\left(1+\frac{1}{n^{2}}\right)\left[\lambda+2 \nu\left(n^{2}+1\right)\right] .
$$

From (4.30), one gets

$$
w_{2}=z_{2} / z_{1}=8(\lambda+4 \nu) .
$$

From (4.5)and (4.31), one has

$$
8(\lambda+4 \nu)=-\frac{1}{a_{2}+\frac{1}{a_{3}+\cdots}} .
$$

(a). $\nu>0$. If $\operatorname{Re}\{\lambda\} \geq 0$, then $\operatorname{Re}\left\{a_{n}\right\}>0$ for $n \geq 2$. Thus the real part of the right hand side of (4.32) is negative, while the real part of its left hand side is positive. Thus there is no eigenvalue of non-negative real part. (b). $\nu=0$. If $\operatorname{Re}\{\lambda\} \geq 0$, then $\operatorname{Re}\left\{a_{n}\right\}(n \geq 2)$ has the same 
fixed sign with $\lambda$, again (4.32) leads to a contradiction. If $\operatorname{Re}\{\lambda\}=0$, let $\tilde{a}=\lim _{n \rightarrow \infty} a_{n}=4 \lambda$, then

$$
\begin{aligned}
& \tilde{a} z_{1}=\frac{1}{2} z_{2}, \\
& \tilde{a} z_{n}=\frac{n^{2}}{n^{2}+1}\left(z_{n+1}-z_{n-1}\right), \quad n \geq 2 .
\end{aligned}
$$

By using $\ell_{2}$ norm of $\left\{z_{n}\right\}_{n \geq 1}$, one sees that possible eigenvalues have to satisfy $|\tilde{a}| \leq 2$. As mentioned before, $\operatorname{Re}\{\lambda\}=0$ and $|\tilde{a}| \leq 2$ correspond to a continuous spectrum $[\mathbf{9}]$, which is the interval $\left[-i \frac{1}{2}, i \frac{1}{2}\right]$. Similarly from (4.29), one gets

$$
w_{-1}=z_{-1} / z_{-2}=-\frac{1}{8(\lambda+4 \nu)} .
$$

From (4.5)and (4.33), one has

$$
-\frac{1}{8(\lambda+4 \nu)}=a_{-2}+\frac{1}{a_{-3}+\frac{1}{a_{-4}+\cdots}} .
$$

Notice that $a_{-n}=a_{n}$, one gets the same conclusion as above. Finally, by choosing $\omega_{n}=0$ if $n \neq 0$, one gets the eigenvalue $\lambda=-2 \nu$ with the eigenfunction

$$
\tilde{\omega}_{\hat{k}} e^{i \hat{k} \cdot x}+\text { c.c. }, \quad \forall \tilde{\omega}_{\hat{k}} \in \mathbb{C} .
$$

(4) In this case,

$$
a_{n}=8 \frac{n^{2}+(n+1)^{2}}{n^{2}+(n+1)^{2}-2}\left\{\lambda+\nu\left[n^{2}+(n+1)^{2}\right]\right\} .
$$

Thus $a_{-(n+1)}=a_{n}$. Equation (4.8) is reduced to

$$
a_{0}+\frac{1}{a_{1}+\frac{1}{a_{2}+\cdots}}= \pm i \text {. }
$$

The first a few $a_{n}$ 's are

$$
\begin{aligned}
& a_{0}=8 \cdot(-1) \cdot(\lambda+\nu), \\
& a_{1}=8 \cdot\left(\frac{5}{3}\right) \cdot(\lambda+5 \nu), \\
& a_{2}=8 \cdot\left(\frac{13}{11}\right) \cdot(\lambda+13 \nu), \\
& a_{3}=8 \cdot\left(\frac{25}{23}\right) \cdot(\lambda+25 \nu) .
\end{aligned}
$$

(a). $\nu>0$. Consider the region $\operatorname{Re}\{\lambda\} \geq-\nu$, in which

$$
\left|a_{n}\right| \geq 8|\lambda+\nu|, \quad \forall n \geq 0 \text {. }
$$

By using $\ell_{2}$ norm of $\left\{z_{n}\right\}_{n \in \mathbb{Z}}$ in (4.3), one sees that possible eigenvalues in this region have to satisfy

$$
|\lambda+\nu| \leq \frac{1}{4}
$$

Next in the region

$$
\mathcal{D}=\left\{\lambda|\operatorname{Re}\{\lambda\} \geq-\nu,| \lambda+\nu \mid \leq \frac{1}{4}\right\}
$$


we will use Rouché's theorem to track the eigenvalues. Let

$$
\begin{aligned}
& f(\lambda)=a_{0}+\frac{1}{a_{1}+\frac{1}{a_{2}+\cdots}}+i, \\
& g(\lambda)=a_{0}+\frac{1}{a_{1}}+i .
\end{aligned}
$$

To apply the Rouché's theorem, one needs to show that

$$
|f(\lambda)-g(\lambda)|<|f(\lambda)|+|g(\lambda)|, \quad \lambda \in \partial \mathcal{D} .
$$

If this is not true, then there is a $\lambda \in \partial \mathcal{D}$ and a $\delta(0 \leq \delta \leq \infty)$ such that

$$
f(\lambda)=-\delta g(\lambda),
$$

i.e.

$$
a_{0}+i+\frac{\frac{1}{1+\delta}}{a_{1}+\frac{1}{a_{2}+\cdots}}+\frac{\delta}{1+\delta} \frac{1}{a_{1}}=0 .
$$

On the part of the boundary $\partial \mathcal{D}: \operatorname{Re}\{\lambda\}=-\nu$,

$$
\operatorname{Re}\left\{a_{0}\right\}=0, \quad \operatorname{Re}\left\{a_{n}\right\}>0, \quad n \geq 1 .
$$

By taking the real part of (4.37), one sees that (4.37) can not be satisfied.

On the other part the boundary $\partial \mathcal{D}:|\lambda+\nu|=\frac{1}{4}$,

$$
\begin{array}{ll}
\left|a_{0}\right|=2, & \left|a_{1}\right| \geq \frac{10}{3}, \quad\left|a_{2}\right| \geq \frac{26}{11}, \\
\left|a_{n}\right| \geq 2, \quad \forall n \geq 3 . &
\end{array}
$$

By a result of continued fraction [15],

$$
\left|\frac{1}{a_{3}+\frac{1}{a_{4}+\cdots}}\right| \leq 1 .
$$

Thus

$$
\begin{aligned}
\mid \frac{\frac{1}{1+\delta}}{a_{1}+\frac{1}{a_{2}+\cdots} \mid} \leq \frac{1}{\left|a_{1}\right|-\left|\frac{1}{a_{2}+\frac{1}{a_{3}+\cdots}}\right|} \\
\leq \frac{1}{\left|a_{1}\right|-\frac{1}{\left|a_{2}\right|-\left|\frac{1}{a_{3}+\frac{1}{a_{4}+\cdots}}\right|}} \\
\leq \frac{1}{\frac{10}{3}-\frac{1}{\frac{26}{11}-1}}=\frac{15}{39} .
\end{aligned}
$$

The rest of (4.37) has the estimate:

$$
\begin{aligned}
& \left|a_{0}+i+\frac{\delta}{1+\delta} \frac{1}{a_{1}}\right| \geq\left|a_{0}\right|-1-\frac{\delta}{1+\delta} \frac{1}{\left|a_{1}\right|} \\
& \geq 2-1-\frac{\delta}{1+\delta} \frac{3}{10} \geq \frac{7}{10} .
\end{aligned}
$$


Therefore, (4.37) can not be satisfied. By Rouché's theorem, inside $\mathcal{D}$, $f(\lambda)$ and $g(\lambda)$ have the same number of roots. $g(\lambda)$ has two roots

$$
\lambda=-3 \nu+\frac{1}{16} i \pm \frac{1}{16} \sqrt{\frac{7}{5}+16(8 \nu)^{2}+i 64 \nu},
$$

and one of which is in $\mathcal{D}$ (at least when $\nu$ is small enough). Thus $f(\lambda)$ has one root in $\mathcal{D}$. From (4.35), this root satisfies

$$
\operatorname{Re}\left\{a_{1}+\frac{1}{a_{0}+i}\right\}<0
$$

which leads to

$$
\begin{gathered}
-\nu<\operatorname{Re}\{\lambda\}<\frac{1}{4} \sqrt{\frac{3}{20}+(8 \nu)^{2}}-2 \nu \\
\frac{1}{8}\left(1-\sqrt{\frac{3}{5}}\right)<\operatorname{Im}\{\lambda\}<\frac{1}{8}\left(1+\sqrt{\frac{3}{5}}\right) .
\end{gathered}
$$

(b). $\nu=0$. Then

$$
\left|a_{n}\right| \geq 8|\lambda|, \quad \forall n \geq 0 .
$$

Again by using $\ell_{2}$ norm of $\left\{z_{n}\right\}_{n \in \mathbb{Z}}$ in (4.3), one sees that possible eigenvalues in this region have to satisfy

$$
|\lambda| \leq \frac{1}{4}
$$

In fact, if there is an eigenvalue with positive real part, then (4.38) is true, which leads to

$$
\begin{gathered}
0<\operatorname{Re}\{\lambda\}<\frac{1}{16} \sqrt{\frac{3}{5}}, \\
\frac{1}{8}\left(1-\sqrt{\frac{3}{5}}\right)<\operatorname{Im}\{\lambda\}<\frac{1}{8}\left(1+\sqrt{\frac{3}{5}}\right) .
\end{gathered}
$$

As proved in $[\mathbf{9}]$, such eigenvalues (if exist) come in quadruplet $(\lambda, \bar{\lambda}-\lambda$ $-\bar{\lambda})$. As in Case 3 above, $\left[-i \frac{1}{4}, i \frac{1}{4}\right]$ is a continuous spectrum $[\mathbf{9}]$.

(5) When $\nu=0$, the union of all the continuous spectra

$$
\left[-i \frac{1}{4}\left|\hat{k}_{2}-\hat{k}_{1}\right|, i \frac{1}{4}\left|\hat{k}_{2}-\hat{k}_{1}\right|\right]
$$

is $i \mathbb{R}$.

4.3. Example 3. An example studied in details by Vincent Liu $[\mathbf{1 3}][\mathbf{1 4}]$ is $\Omega_{*}=-\frac{\sqrt{2}}{\pi} \cos \left(2 x_{2}\right)$ which corresponds to $p=(0,2)^{T}$ and $\Gamma=-\frac{1}{\sqrt{2} \pi}$ in (4.1). Here

$$
\begin{aligned}
a_{n}= & -4 \sqrt{2} \pi \frac{\hat{k}_{1}^{2}+\left(\hat{k}_{2}+2 n\right)^{2}}{\hat{k}_{1}\left[\hat{k}_{1}^{2}+\left(\hat{k}_{2}+2 n\right)^{2}-4\right]} \\
& \times\left\{\lambda+\nu\left[\hat{k}_{1}^{2}+\left(\hat{k}_{2}+2 n\right)^{2}\right]\right\} .
\end{aligned}
$$

TheOREM 4.6. The spectra of the $2 D$ linear Euler operator $L$ have the following properties. 
(1) The set $\{\hat{k}+n p\}_{n \in \mathbb{Z}}$ has no intersection with the disc of radius $|p|=2$. When $\nu>0$, there is no eigenvalue of non-negative real part. When $\nu=0$, the entire spectrum is the continuous spectrum

$$
\left[-i \frac{\left|\hat{k}_{1}\right|}{2 \sqrt{2} \pi}, i \frac{\left|\hat{k}_{1}\right|}{2 \sqrt{2} \pi}\right]
$$

(2) $\hat{k}=(0,1)^{T}$ or $(0,2)^{T}$. The spectrum consists of the eigenvalues

$$
\lambda=-\nu\left[\hat{k}_{1}^{2}+\left(\hat{k}_{2}+2 n\right)^{2}\right], \quad n \in \mathbb{Z},
$$

where for $\hat{k}=(0,2)^{T}, n \neq-1$. The eigenfunctions are the Fourier modes

$$
\tilde{\omega}_{\hat{k}+n p} e^{i(\hat{k}+n p) \cdot x}+\text { c.c. }, \quad \forall \tilde{\omega}_{\hat{k}+n p} \in \mathbb{C}, \quad n \in \mathbb{Z} .
$$

As $\nu \rightarrow 0^{+}$, the eigenvalues are dense on the negative half of the real axis $(-\infty, 0]$. Setting $\nu=0$, the only eigenvalue is $\lambda=0$ of infinite multiplicity with the same eigenfunctions as above.

(3) $\hat{k}=(2,0)^{T}$. When $\nu>0$, there is no eigenvalue of non-negative real part. When $\nu=0$, the entire spectrum is the continuous spectrum $\left[-i \frac{1}{\sqrt{2} \pi}, i \frac{1}{\sqrt{2} \pi}\right]$. A special eigenvalue is $\lambda=-4 \nu$ (when $\nu=0$, this eigenvalue $\lambda=0$ is embedded in the continuous spectrum).

(4) $\hat{k}=(1,0)^{T}$. There is a unique $\nu_{*}$,

$$
\frac{1}{10 \pi} \sqrt{\frac{89}{34}}<\nu_{*}<\frac{1}{10 \pi} \sqrt{3} .
$$

When $\nu>\nu_{*}$, there is no eigenvalue of non-negative real part. When $\nu=\nu_{*}, \lambda=0$ is an eigenvalue, and all the rest eigenvalues have negative real parts. When $\nu<\nu_{*}$, there is a unique positive eigenvalue $\lambda(\nu)>0$, and all the rest eigenvalues have negative real parts. $\nu^{-1} \lambda(\nu)$ is a strictly monotonically decreasing function of $\nu . \lambda(\nu)$ has the estimate

$$
\frac{1}{\pi} \sqrt{\frac{89}{680}}-5 \nu<\lambda(\nu)<\frac{1}{\pi} \sqrt{\frac{3}{20}}-\nu .
$$

In particular, as $\nu \rightarrow 0^{+}, \lambda(\nu)=O(1)$. When $\nu=0$, we have only two eigenvalues $\lambda_{0}$ and $-\lambda_{0}$, where $\lambda_{0}$ is positive

$$
\frac{1}{\pi} \sqrt{\frac{89}{680}}<\lambda_{0}<\frac{1}{\pi} \sqrt{\frac{3}{20}} .
$$

The rest of the spectrum is a continuous spectrum $\left[-i \frac{1}{2 \sqrt{2} \pi}, i \frac{1}{2 \sqrt{2} \pi}\right]$. Moreover,

$$
\lim _{\nu \rightarrow 0^{+}} \lambda(\nu)=\lambda_{0}
$$

(5) $\hat{k}=(1,1)^{T}$. When $\nu>0$, in the half plane $\operatorname{Re}\{\lambda\} \geq-2 \nu$, there is a unique pair of eigenvalues $\lambda$ and $\bar{\lambda}$ such that

$$
\begin{gathered}
-2 \nu<\operatorname{Re}\{\lambda\}<\frac{1}{\pi} \sqrt{\frac{3}{40}}, \\
\frac{\sqrt{5}-\sqrt{3}}{2 \sqrt{10} \pi}<\operatorname{Im}\{\lambda\}<\frac{\sqrt{5}+\sqrt{3}}{2 \sqrt{10} \pi} .
\end{gathered}
$$


When $\nu=0,\left[-i \frac{1}{2 \sqrt{2} \pi}, i \frac{1}{2 \sqrt{2} \pi}\right]$ is a continuous spectrum. If there is an eigenvalue of positive real part, then there is a quadruplet $(\lambda, \bar{\lambda}-\lambda-\bar{\lambda})$ where

$$
\begin{gathered}
0<\operatorname{Re}\{\lambda\}<\frac{1}{\pi} \sqrt{\frac{3}{40}}, \\
\frac{\sqrt{5}-\sqrt{3}}{2 \sqrt{10} \pi}<\operatorname{Im}\{\lambda\}<\frac{\sqrt{5}+\sqrt{3}}{2 \sqrt{10} \pi} .
\end{gathered}
$$

(6) Finally, when $\nu=0$, the union of all the above pieces of continuous spectra is the imaginary axis $i \mathbb{R}$.

REMARK 4.7. Except for Case 4, the rest of the proof is similar to that of Theorem 4.4. Overall, Liu [13] [14] did not realize the continuous spectrum. For Case 4, Liu [13] [14] gave an elegant proof. For Case 5, Liu [13] [14] claimed more than what were actually proved. In [14], the sketch after line 9, pp.472 can be realized as shown in the proof of Case 4 of Theorem 4.4 above. But Equation (39) on the same page does not imply $\operatorname{Re}\left\{\eta_{1}(\nu)\right\}>0$ in Equation (5) on pp.467. Arguments between lines 5 and 8 on pp.483 are not solid, therefore, the existence of $\eta_{1}$ in Part B of Theorem 2 on pp.467 was not proved. The sketch between lines 22 and 25 on pp.484 is not completed, therefore, (14) in Theorem 3 on pp.468 was not proved. Proving these claims seems tricky.

REMARK 4.8. Cases 2 and 3 indicates that $\operatorname{Re}\left\{\lambda_{1}^{s}\right\}$ in Assumption 1 is at least $O(\nu)$ as $\nu \rightarrow 0^{+}$. Case 4 shows the existence of an unstable eigenvalue. In fact, by the property of continued fraction, the corresponding eigenfunction $\Omega^{u}(\nu)$ also converges as $\nu \rightarrow 0^{+}$. Thus $\left\|\Omega^{u}\right\|_{\ell+1} /\left\|\Omega^{u}\right\|_{\ell}$ has a uniform bound as $\nu \rightarrow 0^{+}$.

REMARK 4.9. In an effort to minimize the number of unstable modes to one and to have Case 4 not Case 5 in the above theorem, we need to study the rectangular periodic domain $[0,2 \pi / \alpha] \times[0,2 \pi]$ where $1 / 2<\alpha<1$, and the steady state $\Omega_{*}=$ $\cos x_{2}$. This is our Example 1 studied before.

\section{References}

[1] R. Adams, Sobolev Space, Academic Press, New York, 1975.

[2] L. Bers, F. John, M. Schechter, Partial Differential Equations, Interscience, New York, 1964.

[3] N. Dunford, J. Schwartz, Linear Operators, Part II, Interscience, New York, 1963.

[4] W. Gautschi, Computational aspects of three-term recurrence relations, SIAM Review 9 (1967).

[5] D. Henry, Geometric Theory of Semilinear Parabolic Equations, Lecture Notes in Mathematics, 840, Springer-Verlag, 1981.

[6] W. B. Jones, W. J. Thron, Continued Fractions, Analytic Theory and Applications, Encyclopedia of Math. and Its Appl. 11, 1980.

[7] T. Kato, Quasi-linear equations of evolution, with applications to partial differential equations, Lecture Notes in Math., Springer 448 (1975), 25.

[8] Y. Latushkin, Y. Li, and M. Stanislavova, The spectrum of a linearized 2D Euler operator, Studies in Applied Mathematics 112 (2004), 259.

[9] Y. Li, On 2D Euler equations. I. On the energy-Casimir stabilities and the spectra for linearized 2D Euler equations, J. Math. Phys. 41, no.2 (2000), 728.

[10] Y. Li, On 2D Euler equations: II. Lax pairs and homoclinic structures, Commun. Appl. Nonlinear Analysis 10, no.1 (2003), 1.

[11] Y. Li, Persistent homoclinic orbits for nonlinear Schrödinger equation under singular perturbations, Dynamics of PDE 1, no.1 (2004), 87.

[12] Y. Li, Zero dispersion and viscosity limits of invariant manifolds for focusing nonlinear Schrödinger equations, Submitted to J. Math. Anal. Appl. (2005). 
[13] V. Liu, An example of instability of the Navier-Stokes equations on the 2-dimensional torus, Comm. PDE 17 (1992), 1995.

[14] V. Liu, On unstable and neutral spectra of incompressible inviscid and viscid fluids on the 2D torus, Quart. Appl. Math. LIII, No.3 (1995), 465.

[15] L. Lorentzen, H. Waadeland, Continued Fractions with Applications, North-Holland, Amsterdam, 1992.

[16] L. D. Meshalkin and Ya. G. Sinai, Investigation of the stability of a stationary solution of a system of equations for the plane movement of an incompressible viscous liquid, J. Appl. Math. Mech. (PMM) 25 (1961), 1700.

[17] L. M. Milne-Thomson, The Calculus of Finite Differences, Macmillan, London, 1933.

[18] O. Perron, Über einen Satz der Herren Poincaré, J. Reine Angew. Math. 136 (1910), 17.

[19] O. Perron, Über die Poincarésche lineare Differenzengleichung, J. Reine Angew. Math. 137 (1910), 6.

[20] O. Perron, Uber linear Differenzengleichungen, Acta Math. 34 (1911), 109.

[21] H. Poincaré, Sur les équations linéaires aux différentielles ordinaires et aux différences finies, Amer. J. Math. 7 (1885), 203.

[22] G. Prodi, Teoremi di tipo locale per il sistema di Navier-Stokes e stabilita delle soluzioni stazionarie, Rend. Sem. Mat. Univ. Padova 32 (1962), 374.

[23] M. Reed, B. Simon, Methods Modern Mathematical Physcis, IV, Academic Press, NY, 1978.

[24] D. Sattinger, The mathematical problem of hydrodynamic stability, Journal of Mathematics and Mechanics (Indiana Univ. Math. J.) 19, No.9 (1970), 797.

[25] R. Shvidkoy, Y. Latushkin, The essential spectrum of the linearized 2D Euler operator is a vertical band, Contemp. Math. 327 (2003), 299.

[26] J. Wu, The inviscid limits for individual and statistical solutions of the Navier-Stokes equations, Ph.D. Thesis, Chicago University (1996).

Department of Mathematics, University of Missouri, Columbia, mo 65211

E-mail address: cli@math.missouri.edu 